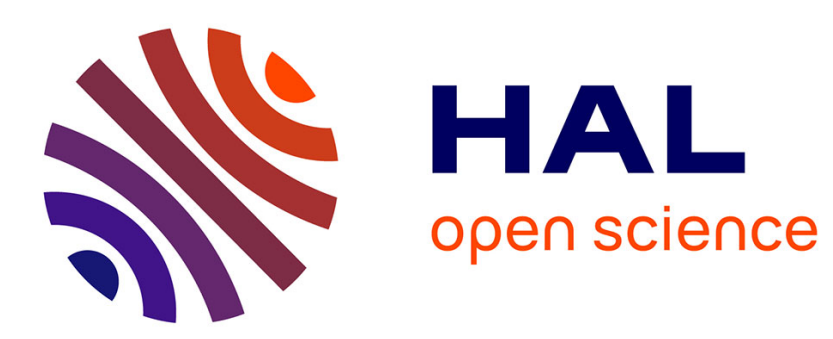

\title{
Stability of variational inequalities and prox-regularity in Hilbert space
}

Matthieu Sebbah

\section{To cite this version:}

Matthieu Sebbah. Stability of variational inequalities and prox-regularity in Hilbert space. 2014. hal-00965285

\section{HAL Id: hal-00965285 \\ https://hal.science/hal-00965285}

Preprint submitted on 25 Mar 2014

HAL is a multi-disciplinary open access archive for the deposit and dissemination of scientific research documents, whether they are published or not. The documents may come from teaching and research institutions in France or abroad, or from public or private research centers.
L'archive ouverte pluridisciplinaire HAL, est destinée au dépôt et à la diffusion de documents scientifiques de niveau recherche, publiés ou non, émanant des établissements d'enseignement et de recherche français ou étrangers, des laboratoires publics ou privés. 


\title{
Stability of variational inequalities and prox-regularity in Hilbert space
}

\author{
M. Sebbah*
}

\begin{abstract}
In this paper we study the problem of stability of solutions of a classical variational inequality in a general Hilbert space. To do so, we use the concepts of local normal map and local natural map to transform the multi-valued problem into a single-valued one. Then we use the degree theory to obtain the stability result under some general hypotheses. Finally we discuss those hypotheses under different concepts of prox-regularity (local and uniform).
\end{abstract}

\section{Introduction}

In several optimization problems, we have to study a recurent variational inequality, $V I_{C, \phi}(u, v, w)$ or $V I(u, v, w)$ when there is no risk of ambiguity, of the form

$$
w \in N(C(u), x)+\phi(v, x)
$$

where $x$ is the unknown variable, $C$ is a set-valued mapping from a topological space $\mathcal{U}$ to a Hilbert space $\mathcal{H}$, $\phi$ is a mapping from $\mathcal{V} \times \mathcal{H}$ to $\mathcal{H}$, with $\mathcal{V}$ another topological space, and $N(C(u), x)$ denotes a certain normal cone of the set $C(u) \subseteq \mathcal{H}$ at the point $x \in \mathcal{H}$.

One well-known example to illustrate the importance of this variational inequality would be that, given a set-valued mapping $C: \mathbb{R} \rightrightarrows \mathbb{R}^{N}$ with non-empty closed convex values and a convex function $\phi: \mathbb{R}^{N} \rightarrow \mathbb{R}$ of class $C^{1}$ then, finding a pair $(x, u) \in \mathbb{R}^{N} \times \mathbb{R}$ such that $x \in \arg \min _{C(u)} \phi$ is equivalent to solving the variational inequality

$$
0 \in \nabla \phi(x)+N(C(u), x)
$$

where $N(C(u), x)$ is the convex normal cone of $C(u)$ at the point $x$. We refer to [9] for several other examples of concrete problems which can be formulated with the variational inequality $(\mathrm{VI}(\mathrm{u}, \mathrm{v}, \mathrm{w}))$. Thus, studying problems of the form $(\mathrm{VI}(\mathrm{u}, \mathrm{v}, \mathrm{w}))$ can be of great interest.

In particular, a relevant question dealing with variational inequalities is the stability of solutions with respect to changes of the parameters. Indeed, considering a set-valued mapping $F: X \rightrightarrows Y$, where $X$ and $Y$ are two topological spaces, and a point $\left(x_{0}, y_{0}\right) \in X \times Y$ satisfying the inclusion $y_{0} \in F\left(x_{0}\right)$, it is crucial to know whether, for $x$ sufficiently close to $x_{0}$, the inclusion $y_{0} \in F(x)$ still holds, or symmetrically, for $y$ sufficiently close to $y_{0}$, the inclusion $y \in F\left(x_{0}\right)$ is satisfied.

In the single valued case, i.e., of the form $y=F(x)$ with $F$ a mapping instead of a set-valued mapping, one of the fundamental tools to study existence and stability of solutions is given by the degree theory. This is the reason why great efforts have been made to reduce set-valued problems into single-valued ones.

Such a reduction has been realized by Robinson in [18] for the study of the variational inequality

$$
w \in N(C(u), x)+\phi(u, x)
$$

in the finite dimensional setting, i.e., keeping the same notations than in $(\mathrm{VI}(\mathrm{u}, \mathrm{v}, \mathrm{w}))$ with $\mathcal{H}=\mathbb{R}^{N}$ with the difference that, in Robinson's equation, the mapping $\phi$ is defined on $\mathcal{U} \times \mathcal{H}$, whereas in $(\mathrm{VI}(\mathrm{u}, \mathrm{v}, \mathrm{w})), \phi$ is

\footnotetext{
${ }^{* 1}$ Universidad Tecnica Federico Santa Maria, Departamento de matematica, Avenida Espana 1680, Valparaiso, Chile ; INRIA Chile, CIRIC project, team Bionature. E-mail: matthieu.sebbah@usm.cl
} 
defined on $\mathcal{V} \times \mathcal{H}$. To study the stability of the solutions, Robinson in [16, 17] generalized the approach used in the convex setting (i.e. $C$ convex valued) and introduced the concept of localized normal map. He showed that under the "right assumptions" on $C$ and $\phi$, the set of solutions $(u, w, x)$ satisfying (Rob) is topologically equivalent to the set of solutions $(u, w, z)$ satisfying an equation of the form

$$
w=f(u, z)
$$

$f$, defined on a subset of $\mathcal{U} \times \mathbb{R}^{N}$, being the so called normal map associated to (Rob). Thus he transformed a multivalued problem (Rob) into a single valued one (1). Then he used the degree theory to solve the stability problem on (1) and, by the topological equivalence between the solutions sets of (Rob) and (1), he obtained stability results on (Rob).

One of the main interest of Robinson's work is that he showed in [19] that, in the finite dimensional setting, those "right assumptions" needed to obtain the topological equivalence between (Rob) and (1) are fulfilled by a class of set-valued mappings larger than the class of non-empty closed convex valued set-valued mappings. Those set-valued mappings are called prox-regular with compatible parametrization and it is well known that the concept of prox-regularity of sets is a good candidate to generalize properties from the convex setting based on the uniqueness of the projection mapping in Hilbert spaces (see [2], [3], [5], [7], [14], [20] and references therein).

In a previous work, see [21], we generalized Robinson's work in [19] to the setting of general Hilbert space in showing that prox-regular set-valued mapping satisfy also the "right assumptions" in this general context. This inclines us to pursue the generalization of Robinson's results to the possibly infinite dimensional Hilbert spaces. This paper aims to establish stability results of $(\mathrm{VI}(\mathrm{u}, \mathrm{v}, \mathrm{w}))$ in the general Hilbert setting through the method used by Robinson in [18].

Our work is also highly inspired by Kien and Yao's paper [10]. These authors used Robinson's method to study, in the general reflexive Banach space setting, the stability of the solutions of the variational inequality

$$
0 \in N(C(u), x)+\phi(v, x)
$$

using the same notation than in $(\mathrm{VI}(\mathrm{u}, \mathrm{v}, \mathrm{w}))$, forgetting about the dependance of $w$ involved in $(\mathrm{VI}(\mathrm{u}, \mathrm{v}, \mathrm{w}))$. However, they do not use Robinson's localized normal map, but what they call generalized normal maps in order to turn the multivalued problem into a single-valued one. Then, they cite Robinson's stability result as a corollary of their result to the finite dimensional case, but, strictly speaking, it is not fully a corollary for two reasons. First to get Robinson's result, we have to follow Kien and Yao's proof with the localized normal map instead of the genralized normal map. The second reason is that there are no a priori links between the degree of the localized normal map and the generalized one, so we cannot deduce Robinson's result under Kien and Yao's hypothesis. However, the method in KY shows that a generalized abstract feature can be established through the degree theory in view of obtaining both Robinson's stability result and Kien and Yao's one.

The present paper provides, in our view, three main contributions. The first one is that it unifies the inequalities studied by Robinson and Kien and Yao, in the sense that, (Rob) and (KY) can both be deduced from $(\mathrm{VI}(\mathrm{u}, \mathrm{v}, \mathrm{w}))$. The second contribution is the generalization in a more general framework of the proof of Robinson's and Kien and Yao's stability results using degree theory. And the third interesting point is that our results are valid for a large class of set-valued mappings that may not be convex-valued in the general Hilbert setting.

We organize our work as follows. In the second section, we start by shortly recalling some features of degree theory in order to give a general abstract stability result using tools of degree theory. In the third section, we define the concept of local normal map and see that, under some basic key hypotheses, the set of solutions of the variational inequality $(\mathrm{VI}(\mathrm{u}, \mathrm{v}, \mathrm{w}))$ and the set of solutions of the local normal map are topologically equivalent. This allows us to apply the stability result of section 2 to get stability results of solutions of $(\mathrm{VI}(\mathrm{u}, \mathrm{v}, \mathrm{w}))$. Finally, we show in the third section that the class of prox-regular set-valued mappings satisfies one of those key hypothesis needed to have the topological equivalence shown in the third section.

\section{$2 \quad$ A stability result using degree theory}

In this section, our purpose is to study the stability of solutions of equations of the form $f(t, \cdot)=y_{0}$, where $t$ is a more general variable than $u$, on a set $\Omega$ with a variation on $t$. Consider a topological space $\mathcal{T}$, a Banach space $\mathcal{E}$, a subset $\Omega$ of $\mathcal{E}$ and a mapping $f: \mathcal{T} \times \mathcal{E} \rightarrow \mathcal{E}$. Given $y_{0} \in \mathcal{E}$ and $t_{0} \in \mathcal{T}$, a first interesting 
question is to know whether the equation $f\left(t_{0}, \cdot\right)=y_{0}$ has a solution in $\Omega$, that is, to know whether there exists a certain $x_{0} \in \Omega$ such that $f\left(t_{0}, x_{0}\right)=y_{0}$. Assuming that such a solution exists, the second question that comes naturally in mind is the stability of the solution on $\Omega$ with a variation on $t$, i.e., if $t$ is "close to" $t_{0}$, the equation $f(t, \cdot)=y_{0}$ still has a solution on $\Omega$. The intuition would tell us that if the function $f$ is not "too bad", for instance if $f$ is continuous on $\mathcal{T} \times \mathcal{E}$, then $f(t, \cdot)$ being " close to " $f\left(t_{0}, \cdot\right)$, we could expect that $f(t, \cdot)=y_{0}$ has also a solution in $\Omega$. Unfortunately, this is not enough, as we can see with the very simple following example. Considering

$$
\begin{aligned}
f: \mathbb{R} \times \mathbb{R} & \rightarrow \mathbb{R} \\
(t, x) & \mapsto x^{2}+t
\end{aligned}
$$

we notice that the equation $f(0, \cdot)=0$ has a (unique) solution on $\Omega=]-1 ; 1[$, which is $x=0$. However, as soon as $t>0$, the equation $f(t, \cdot)=0$ does not have any solution on $]-1 ; 1[$.

The main reason of this lack of stability of the solution is that the topological degree of the function $f(0, \cdot)$ on $\Omega=$ ] $-1 ; 1$ [ for the point $y_{0}=0$ is equal to zero. To understand it, we first recall the topological degree of a mapping on a set for a certain point.

\subsection{Topological degree}

As the aim of this paper is not to give a lecture on the topological degree, we will only present the results that will be used later on without giving any proof. However, the interested reader would find those proofs in [6] (very good and in French) and [8] from which we have been highly inspired.

Considering a mapping $\varphi$ from a Banach space $\mathcal{E}$ into itself, an open subset $\Omega$ of $\mathcal{E}$ and a point $y_{0}$ of $\mathcal{E}$, the degree aims to answer the question: is there a solution to the equation $\varphi(\cdot)=y_{0}$ that belongs to $\Omega$ ? A good way to compare the degree of a mapping in the finite dimensional setting is to consider the determinant of a linear mapping $\varphi$ of a finite dimensional vector space $\mathcal{E}$ into itself. If the determinant is non equal to zero, then the map is onto and so for every $y_{0} \in \mathcal{E}$, there exists $x_{0} \in \mathcal{E}$ satisfying $\varphi\left(x_{0}\right)=y_{0}$. Roughly speaking, the degree acts in the same way adding the constraint that $x_{0}$ has to belong to a given subset $\Omega$ of $\mathcal{E}$. Thus, if $\operatorname{deg}\left(\varphi, \Omega, y_{0}\right)$, the degree of the triple $\left(\varphi, \Omega, y_{0}\right)$ is non equal to zero, there will exist $x_{0} \in \Omega$ such that $\varphi\left(x_{0}\right)=y_{0}$.

However, all the solutions of $\varphi(\cdot)=y_{0}$ in $\Omega$ are not necessarily taken into account by the degree. Indeed, one of the principal property of the degree is that it is stable under certain homotopies (cf. 3) of Definition $2.1)$. Thus, if $\psi$ is such a homotopy of $\varphi$, then $\operatorname{deg}\left(\varphi, \Omega, y_{0}\right)=\operatorname{deg}\left(\psi, \Omega, y_{0}\right)$. This highlights the reason for which our previous example, $f(t, x)=x^{2}+t \operatorname{did}$ not work. Indeed, for $t=0$, we consider $\varphi(x)=f(0, x)=x^{2}$ which does have $x=0$ as a solution for the equation $\varphi(\cdot)=0$ on $]-1,1$. However, this solution is not taken into account by the degree, i.e., $\operatorname{deg}(\varphi]-,1 ; 1[, 0)=0$, because there exists a a homotopy $\psi$ such that $\psi(\cdot)=0$ does not have any solution on on $]-1 ; 1[($ take $\psi(x)=f(t, x)$ with $t>0)$.

Moreover, as the determinant is defined only for the class of linear mappings, the degree is not defined for any mapping. In fact, there exist many ways to define degree concepts that differ mainly on the considered class of functions. For our part, we will first introduce the Brouwer's degree for continuous functions defined on finite dimensional spaces, then we will present the Leray-Schauder's degree defined on continuous perturbations with finite range of the identity map in any Banach space.

\subsubsection{The Brouwer's degree in finite dimensional space}

In this subsection, $\mathcal{E}$ will be a finite-dimensional real vector space of dimension $N$ endowed with any of the usual norms, $\Omega$ will be an open and bounded subset of $\mathcal{E}, y_{0}$ a point in $\mathcal{E}$ and $\varphi: \bar{\Omega} \rightarrow \mathcal{E}$ a continuous mapping from the closure of $\Omega$ to $\mathcal{E}$ such that $y_{0} \notin \varphi($ bd $\Omega)$ with bd $\Omega$ being the boundary of $\Omega$. We will denote by $\mathcal{A}_{\mathcal{E}}$ the set of triples $\left(\varphi, \Omega, y_{0}\right)$ satisfying the previous properties (the subscript $\mathcal{E}$ will become important in the next subsection where we will consider several vector spaces). We use the following characterization of the degree as a defintion (cf $[6])$.

Definition 2.1. There exists a unique function $\operatorname{deg}_{\mathcal{E}}: \mathcal{A}_{\mathcal{E}} \rightarrow \mathbb{Z}$ called the Brouwer's degree such that:

1) (normalisation) if $\Omega$ is an open and bounded subset of $\mathcal{E}$ and $y_{0} \in \Omega$, then

$$
\operatorname{deg}_{\mathcal{E}}\left(I, \Omega, y_{0}\right)=1
$$

where $I$ denotes the identity map of $\mathcal{E}$; 
2) (additivity) if $\left(\varphi, \Omega, y_{0}\right) \in \mathcal{A}_{\mathcal{E}}$ and $\Omega_{1}$ and $\Omega_{2}$ are two open subsets of $\Omega$ such that $y_{0} \notin \varphi\left(\bar{\Omega} \backslash\left(\Omega_{1} \cup \Omega_{2}\right)\right)$, then

$$
\operatorname{deg}_{\mathcal{E}}\left(\varphi, \Omega, y_{0}\right)=\operatorname{deg}_{\mathcal{E}}\left(\varphi, \Omega_{1}, y_{0}\right)+\operatorname{deg}_{\mathcal{E}}\left(\varphi, \Omega_{2}, y_{0}\right)
$$

3) (homotopy invariance) if $\Omega$ is an open bounded subset of $\mathcal{E}$, and $h:[0 ; 1] \times \bar{\Omega} \rightarrow \mathcal{E}$ and $y:[0,1] \rightarrow \mathcal{E}$ are two continuous mappings such that $y(\lambda) \notin h(\lambda, \operatorname{bd} \Omega)$ for all $\lambda \in[0 ; 1]$, then

$$
\operatorname{deg}_{\mathcal{E}}(h(0, \cdot), \Omega, y(0))=\operatorname{deg}_{\mathcal{E}}(h(1, \cdot), \Omega, y(1)) .
$$

Then, we have the most important property of the degree (see [6]).

Proposition 2.2. Let $\left(\varphi, \Omega, y_{0}\right) \in \mathcal{A}_{\mathcal{E}}$. If $\operatorname{deg}_{\mathcal{E}}\left(\varphi, \Omega, y_{0}\right) \neq 0$, then there exists $x_{0} \in \Omega$ such that $\varphi\left(x_{0}\right)=y_{0}$.

We will also need the following property (see Theorem 2.7 of [8]).

Proposition 2.3 (compact excision property). Let $\left(\varphi, \Omega, y_{0}\right) \in \mathcal{A}_{\mathcal{E}}$ and $K$ be a compact subset of $\bar{\Omega}$ such that $y_{0} \notin \varphi(K)$, then

$$
\operatorname{deg}_{\mathcal{E}}\left(\varphi, \Omega, y_{0}\right)=\operatorname{deg}_{\mathcal{E}}\left(\varphi, \Omega \backslash K, y_{0}\right) .
$$

Now we see how the Brouwer's degree can be extended to the infinite dimensional setting.

\subsubsection{The Leray-Schauder's degree in infinite dimensional space}

To be able to talk about the degree in infinite dimensional spaces, we first need to introduce the notion of degree of a mapping on a vector subspace.

Let us consider a finite dimensional space $\mathcal{E}$ and $\mathcal{F}$ a vector subspace of $\mathcal{E}$. As above, we will denote by $\mathcal{A}_{\mathcal{E}}$ the set of triples $\left(\varphi, \Omega, y_{0}\right)$ such that $\Omega \subseteq \mathcal{E}$ is open and bounded, $\varphi: \bar{\Omega} \rightarrow \mathcal{E}$ is continuous, and $y_{0} \notin \varphi($ bd $\mathcal{E} \Omega)$, and we will use $\mathcal{A}_{\mathcal{F}}$ for the set of triples $\left(\psi, \Theta, z_{0}\right)$ such that $\Theta \subseteq \mathcal{F}$ is open in $\mathcal{F}$ and bounded, $\psi: \bar{\Theta} \rightarrow \mathcal{F}$ is continuous and $z_{0} \notin \psi\left(\mathrm{bd}_{\mathcal{F}} \Theta\right)$.

Assume now that $\Omega \subseteq \mathcal{E}$ is open in $\mathcal{E}$ and bounded as always but that $\varphi$, defined on $\bar{\Omega}$, takes its values in $\mathcal{F}$ instead of $\mathcal{E}$ and that $y_{0} \in \mathcal{F} \backslash \varphi\left(\operatorname{bd}_{\mathcal{E}} \Omega\right)$. As $\mathcal{F} \subseteq \mathcal{E}$, we have $\left(\varphi, \Omega, y_{0}\right) \in \mathcal{A}_{\mathcal{E}}$, so $\operatorname{deg}_{\mathcal{E}}\left(\varphi, \Omega, y_{0}\right)$ is well defined. Moreover, $\Omega \cap \mathcal{F}$ is open in $\mathcal{F}$ and bounded, the restriction $\left.\varphi\right|_{\overline{\Omega \cap \mathcal{F}}}$ of $\varphi$ to $\overline{\Omega \cap \mathcal{F}}$ is continuous, and $y_{0} \notin \varphi(\overline{\Omega \cap \mathcal{F}} \backslash \Omega \cap \mathcal{F})$, hence $\left(\left.\varphi\right|_{\overline{\Omega \cap \mathcal{F}}}, \Omega \cap \mathcal{F}, y_{0}\right) \in \mathcal{A}_{\mathcal{F}}$ and so we can talk about $\operatorname{deg}_{\mathcal{F}}\left(\left.\varphi\right|_{\overline{\Omega \cap \mathcal{F}}}, \Omega \cap \mathcal{F}, y_{0}\right)$.

A fair question is to know whether

$$
\operatorname{deg}_{\mathcal{E}}\left(\varphi, \Omega, y_{0}\right)=\operatorname{deg}_{\mathcal{F}}\left(\left.\varphi\right|_{\Omega \cap \mathcal{F}}, \Omega \cap \mathcal{F}, y_{0}\right) .
$$

The next proposition provides a partial response. It says that for continuous mappings of the form

$$
\varphi=I-\psi
$$

with $\varphi: \bar{\Omega} \rightarrow \mathcal{E}$ and $\psi: \bar{\Omega} \rightarrow \mathcal{F}$ continuous, then if $x_{0} \in \varphi^{-1}\left(y_{0}\right)$ with $y_{0} \in \mathcal{F} \backslash \varphi($ bd $\Omega)$, we have $x_{0}=$ $y_{0}+\psi\left(x_{0}\right) \in \mathcal{F}$, and the desired equality (2) holds true. This leads us to the following property (cf. [6]).

Proposition 2.4. Let $\mathcal{E}$ be a finite dimensional vector space, $\mathcal{F}$ be a vector subspace of $\mathcal{E}, \Omega$ be an open and bounded subset of $\mathcal{E}, \varphi: \bar{\Omega} \rightarrow \mathcal{F}$ be a continuous mapping and $y_{0} \in \mathcal{F} \backslash(I-\varphi)(\mathrm{bd} \Omega)$.

Then

$$
\operatorname{deg}_{\mathcal{E}}\left(I-\varphi, \Omega, y_{0}\right)=\operatorname{deg}_{\mathcal{F}}\left(\left.(I-\varphi)\right|_{\overline{\Omega \cap \mathcal{F}}}, \Omega \cap \mathcal{F}, y_{0}\right) .
$$

We can now generalize the Brouwer's degree to the infinite dimensional case by modifying once again the class of involved mappings.

Let $\mathcal{E}$ be any Banach space and $\Omega$ be an open bounded subset of $\mathcal{E}$. The idea is to consider continuous perturbations with finite dimensional range of the identity map, i.e., of the form $I-\varphi$ where $\varphi: \bar{\Omega} \rightarrow \mathcal{E}$ is such that $\varphi(\bar{\Omega})$ is contained in a finite dimensional vector subspace of $\mathcal{E}$. Thus, considering $y_{0} \notin(I-\varphi)($ bd $\Omega)$ and a finite dimensional vector subspace $\mathcal{F}$ of $\mathcal{E}$ such that $\varphi(\bar{\Omega}) \subseteq \mathcal{F}$ and $y_{0} \in \mathcal{F}$, we can talk about $\operatorname{deg}_{\mathcal{F}}\left(\left.(I-\varphi)\right|_{\overline{\Omega \cap \mathcal{F}}}, \Omega \cap \mathcal{F}, y_{0}\right)$. By Proposition 2.4, considering any other finite dimensional subspace $\mathcal{G}$ of $\mathcal{E}$ such that $\varphi(\bar{\Omega}) \subseteq \mathcal{G}$ and $y_{0} \in \mathcal{G}$, we have

$$
\operatorname{deg}_{\mathcal{G}}\left(\left.(I-\varphi)\right|_{\overline{\Omega \cap \mathcal{G}}}, \Omega \cap \mathcal{G}, y_{0}\right)=\operatorname{deg}_{\mathcal{F}}\left(\left.(I-\varphi)\right|_{\overline{\Omega \cap \mathcal{F}}}, \Omega \cap \mathcal{F}, y_{0}\right)
$$

This number being independent of the considered vector subspace, we can finally define a degree on $\mathcal{E}$ as follows. 
Definition 2.5. Let $\mathcal{E}$ be a Banach space, $\Omega \subseteq \mathcal{E}$ be an open bounded subset, $\varphi: \bar{\Omega} \rightarrow \mathcal{E}$ be a continuous mapping with finite dimensional range (i.e. $\varphi(\bar{\Omega})$ is contained in a finite dimensional vector subspace of $\mathcal{E}$ ) and $y_{0} \notin(I-\varphi)(\mathrm{bd} \Omega)$.

We define the Leray-Schauder degree by

$$
\operatorname{deg}_{\mathcal{E}}\left(I-\varphi, \Omega, y_{0}\right)=\operatorname{deg}_{\mathcal{F}}\left(\left.(I-\varphi)\right|_{\overline{\Omega \cap \mathcal{F}}}, \Omega \cap \mathcal{F}, y_{0}\right),
$$

where $\mathcal{F}$ is any finite dimensional vector subspace of $\mathcal{E}$ such that $\varphi(\bar{\Omega}) \subseteq \mathcal{F}$ and $y_{0} \in \mathcal{F}$.

We have finally presented all the tools that will be needed to obtain our stability result.

\section{$2.2 \quad$ A stability result}

Let us remember that our goal is to study stability of solutions of equations of the form $f(t, \cdot)=y_{0}$ on a given set $\Omega$ under variations of $t$, where $\mathcal{T}$ is a topological space, $\mathcal{E}$ is a Banach space, $f: \mathcal{T} \times \mathcal{E} \rightarrow \mathcal{E}$ is a continuous mapping, $\Omega$ is an open bounded subset of $\mathcal{E}$ and $y_{0} \in \mathcal{E}$.

Before giving the result, we recall the definition of the lower semicontinuity of a set-valued mapping at a given point, as it appears in Theorem 2.7 below.

Definition 2.6. A set-valued mapping $F$ from a topological space $T$ to another topological space $Y$ is said to be lower semicontinuous at a point $t_{0} \in T$ if for all open set $O$ of $Y$ such that $F\left(t_{0}\right) \cap O \neq \emptyset$, there exists a neighbourhood $V$ of $t_{0}$ such that $F(t) \cap O \neq \emptyset$, for every $t \in V$.

We can now present the stability result.

Theorem 2.7. Let $f: \mathcal{T} \times \mathcal{E} \rightarrow \mathcal{E}, \Omega \subseteq \mathcal{E}$ be a nonempty open bounded subset of $\mathcal{E}$ and $V \subseteq \mathcal{T}$ be an open set such that $f$ is continuous on $V \times \bar{\Omega}$ and for every $(t, x) \in V \times \bar{\Omega}, f$ can be decomposed as $f(t, x)=x-g(t, x)$ where $g: V \times \bar{\Omega} \rightarrow \mathcal{E}$ is with finite dimensional range. Let $t_{0} \in V$ and $y_{0} \in \mathcal{E}$ be such that $y_{0} \notin f\left(t_{0}\right.$, bd $\left.\Omega\right)$.

Consider the set-valued mapping

$$
\begin{aligned}
F: \mathcal{T} & \rightrightarrows \mathcal{E}, \\
t & \mapsto f^{-1}(t, \cdot)\left\{y_{0}\right\} \cap \bar{\Omega}
\end{aligned}
$$

and suppose

(i) $F\left(t_{0}\right)$ is a singleton, say $F\left(t_{0}\right)=\left\{x_{0}\right\}$;

(ii) $\operatorname{deg}_{\mathcal{E}}\left(f\left(t_{0}, \cdot\right), \Omega, y_{0}\right) \neq 0$.

Then $F$ takes on nonempty values on a neighbourhood of $t_{0}$ and is lower semicontinuous at the point $t_{0}$.

Proof. Let $O$ be an open subset of $\mathcal{E}$ such that $F\left(t_{0}\right) \cap O \neq \emptyset$, i.e., with $x_{0} \in O$. Let $\mathcal{F}$ be a finite dimensional vector subspace of $\mathcal{E}$ such that $g(V \times \bar{\Omega}) \subseteq \mathcal{F}$ and $y_{0} \in \mathcal{F}$ and set $\Omega_{\mathcal{F}}:=\Omega \cap \mathcal{F}$. We have by definition of the Leray-Schauder degree that

$$
0 \neq \operatorname{deg}_{\mathcal{E}}\left(f\left(t_{0}, \cdot\right), \Omega, y_{0}\right)=\operatorname{deg}_{\mathcal{F}}\left(\left.f\left(t_{0}, \cdot\right)\right|_{\overline{\Omega_{\mathcal{F}}}}, \Omega_{\mathcal{F}}, y_{0}\right) .
$$

Now consider $O_{\mathcal{F}}:=O \cap \Omega_{\mathcal{F}}=O \cap \Omega \cap \mathcal{F}$. Notice that, as $y_{0} \notin f\left(t_{0}\right.$, bd $\Omega$ ) and by (i), we have $x_{0} \in O \cap \Omega$ with $x_{0}-g\left(t_{0}, x_{0}\right)=f\left(t_{0}, x_{0}\right)=y_{0} \in \mathcal{F}$. Thus $x_{0}=g\left(t_{0}, x_{0}\right)+y_{0} \in \mathcal{F}$, and $x_{0} \in O_{\mathcal{F}}$ which implies $O_{\mathcal{F}} \neq \emptyset$. Moreover, as $O \cap \Omega$ is an open subset of $\mathcal{E}, O_{\mathcal{F}}$ is open in $\mathcal{F}$ and so $\overline{\Omega_{\mathcal{F}}} \backslash O_{\mathcal{F}}$ is closed and bounded in the finite dimensional space $\mathcal{F}$, therefore $\overline{\Omega_{\mathcal{F}}} \backslash O_{\mathcal{F}}$ is compact in $\mathcal{F}$.

Also as $F\left(t_{0}\right)=\left\{x_{0}\right\}$ with $x_{0} \in O_{\mathcal{F}}$, we have $y_{0} \notin f\left(t_{0}, \cdot\right)\left(\overline{\Omega_{\mathcal{F}}} \backslash O_{\mathcal{F}}\right)$. We can then apply the excision property of a compact subset to obtain

$$
\operatorname{deg}_{\mathcal{F}}\left(\left.f\left(t_{0}, \cdot\right)\right|_{\overline{O_{\mathcal{F}}}}, O_{\mathcal{F}}, y_{0}\right)=\operatorname{deg}_{\mathcal{F}}\left(\left.f\left(t_{0}, \cdot\right)\right|_{\overline{\Omega_{\mathcal{F}}}}, \Omega_{\mathcal{F}}, y_{0}\right) \neq 0
$$

Now, since $x_{0}$ is the unique point in $\bar{\Omega}$ satisfying $f\left(t_{0}, x_{0}\right)=y_{0}$ and since bd $O_{\mathcal{F}} \subseteq \bar{\Omega}$, we deduce that $f\left(t_{0}, x\right) \neq y_{0}$, for all $x \in \operatorname{bd} O_{\mathcal{F}}$. Thus, for each $x \in \operatorname{bd} O_{\mathcal{F}}$, there exists $\delta_{x}>0$ such that

$$
y_{0} \notin B\left(f\left(t_{0}, x\right), \delta_{x}\right)=: B_{x},
$$


where $B\left(f\left(t_{0}, x\right), \delta_{x}\right)$ denotes the open ball of radius $\delta_{x}$ centered at $f\left(t_{0}, x\right)$. Therefore, by continuity of $f$ on $V \times \bar{\Omega}$, for each $x \in \partial O_{\mathcal{F}}$, there exist a neighbourhood $V_{x} \subseteq V$ of $t_{0}$ and a neighbourhood $X_{x} \subseteq \bar{\Omega}$ of $x$ such that $f\left(V_{x} \times X_{x}\right) \subseteq B_{x}$.

Moreover, since bd $O_{\mathcal{F}}$ is compact (closed and bounded) in the finite dimensional space $\mathcal{F}$, and since $\left\{X_{x} \cap \mathcal{F}\right\}_{x \in \partial O_{\mathcal{F}}}$ is an open cover of bd $O_{\mathcal{F}}$, there exist $x_{1}, \ldots, x_{n} \in \operatorname{bd} O_{\mathcal{F}}$ such that

$$
\operatorname{bd} O_{\mathcal{F}} \subseteq \bigcup_{i=1}^{n} X_{x_{i}} \cap \mathcal{F} .
$$

Let us consider the neighbourhood $V=\cap_{i=1}^{n} V_{x_{i}}$ of $t_{0}$ and let us show that $F(t) \cap O \neq \emptyset$, for all $t \in V$. Let $t \in V$ and let

$$
\begin{aligned}
h:[0 ; 1] \times \overline{O_{\mathcal{F}}} & \rightarrow E \\
(\lambda, x) & \mapsto \lambda f\left(t_{0}, x\right)+(1-\lambda) f(t, x)
\end{aligned}
$$

be the natural homotopy between $f\left(t_{0}, \cdot\right)$ and $f(t, \cdot)$ on $\overline{O_{\mathcal{F}}}$. We show that $y_{0} \neq h(\lambda, x)$, for all $x \in$ bd $O_{\mathcal{F}}$, in order to apply the homotopy invariance of the degree. Let $x \in \operatorname{bd} O_{\mathcal{F}}$. Since $\operatorname{bd} O_{\mathcal{F}} \subseteq \cup_{i=1}^{n} X_{x_{i}} \cap \mathcal{F}$, there exists $i \in\{1, \ldots, n\}$ such that $x \in X_{x_{i}} \cap \mathcal{F}$ and since $t_{0}$ and $t$ are in $V$, we have that $(t, x) \in V_{x_{i}} \times X_{x_{i}}$, hence $f\left(t_{0}, x\right)$ and $f(t, x)$ are in $B_{x_{i}}$. Now, as $B_{x_{i}}$ is convex, we get that for all $\lambda \in[0 ; 1]$,

$$
h(\lambda, x)=\lambda f\left(t_{0}, x\right)+(1-\lambda) f(t, x) \in B_{x_{i}} .
$$

But we saw that $y_{0} \notin B_{x_{i}}$, so for all $x \in \operatorname{bd} O_{\mathcal{F}}$ and for all $\lambda \in[0 ; 1]$, we have $h(\lambda, x) \neq y_{0}$. We can then apply the homotopy invariance of the degree to obtain that

$$
\operatorname{deg}_{\mathcal{F}}\left(\left.f(t, \cdot)\right|_{\overline{O_{\mathcal{F}}}}, O_{\mathcal{F}}, y_{0}\right)=\operatorname{deg}_{\mathcal{F}}\left(\left.f\left(t_{0}, \cdot\right)\right|_{\overline{O_{\mathcal{F}}}}, O_{\mathcal{F}}, y_{0}\right) \neq 0
$$

Therefore by Proposition 2.2, we have that, for all $t \in V$, there exists $x \in O_{\mathcal{F}}$ such that $f(t, x)=y_{0}$, and since $O_{\mathcal{F}} \subseteq O \cap \Omega$, we get that for all $t \in V, F(t) \cap O \neq \emptyset$, and also $F(t) \neq \emptyset$. This concludes the proof.

We see now how this result can be applied to solve stability problems of variational equalities through the concepts of normal and natural mappings.

\section{Stability of solutions of variational inequalities through localiza- tion of normal and natural maps}

As it is already mentioned in the introduction, the aim of the paper is to study the stability of solutions of variational inequalities of the form

$$
w \in N(C(u), x)+\phi(v, x) .
$$

To do so, we will transform the multi-valued problem into a single valued one, using Robinson's local normal map and Kien and Yao's local natural map.

Throughout this section $\mathcal{U}, \mathcal{V}$ will be two topological spaces, $\mathcal{H}$ a Hilbert space, $\phi: \mathcal{V} \times \mathcal{H} \rightarrow \mathcal{H}$ a mapping and $C: \mathcal{U} \rightrightarrows \mathcal{H}$ a non-empty closed valued set-valued mapping. We also consider a normal cone concept which associates to a set $S \subseteq \mathcal{H}$ and a point $x \in S$ a cone $N(S, x) \subseteq \mathcal{H}$ in such a way that $N(S, x)$ coincides with the normal cone in the sense of Convex Analysis whenever the set $S$ is convex.

\subsection{Local normal and natural maps}

To introduce the concepts of local normal and natural maps, let us assume for the moment that $C(\cdot)$ is convex valued. We first remark that the variational inequality

$$
w \in N(C(u), x)+\phi(v, x)
$$

can be rewritten as

$$
x \in(N(C(u), \cdot)+I)^{-1}(w-\phi(v, x)+x) .
$$


We know, by convexity of $C(u)$, that the set-valued mapping

$$
\begin{aligned}
\Pi: \mathcal{U} \times \mathcal{H} & \rightrightarrows \mathcal{H} \\
(u, z) & \mapsto(N(C(u), \cdot)+I)^{-1}
\end{aligned}
$$

is in fact single-valued and equal to the projection mapping, i.e., for all $(u, z) \in \mathcal{U} \times \mathcal{H}$,

$$
\Pi(u, z)=P_{C(u)}(z),
$$

where $P_{C(u)}(z)$ denotes the unique nearest point in $C(u)$ of $z$. This is due to the fact that $N(C(u), \cdot)$ coincides with the normal cone of $C(u)$ in the sense of Convex Analysis.

Thus (3) is equivalent to

$$
x=P_{C(u)}(w-\phi(v, x)+x),
$$

i.e.,

$$
0=x-P_{C(u)}(w-\phi(v, x)+x),
$$

or also, by the change of variable $z=w-\phi(v, x)+x$, equivalent to,

$$
z-w+\phi\left(v, P_{C(u)}(z)\right)=P_{C(u)}(z),
$$

i.e.,

$$
w=z-P_{C(u)}(z)+\phi\left(v, P_{C(u)}(z)\right) .
$$

Thus, defining the normal map as,

$$
f:(u, v, z) \rightarrow z-P_{C(u)}(z)+\phi\left(v, P_{C(u)}(z)\right),
$$

and the natural map as,

$$
g:(u, v, w, x) \rightarrow x-P_{C(u)}(w-\phi(v, x)+x),
$$

we have turned the multi-valued problem $(\mathrm{VI}(\mathrm{u}, \mathrm{v}, \mathrm{w}))$ into two single-valued ones: one using the variable $z$,

$$
f(u, v, z)=w,
$$

(norm.)

the other using the variable $x$,

$$
g(u, v, w, x)=0 .
$$

Note here, that for fixed $u_{0}, v_{0}, w_{0}$, the maps $f\left(u_{0}, v_{0}, \cdot\right)$ and $g\left(u_{0}, v_{0}, w_{0}, \cdot\right)$ are both perturbations of the identity map, which will allow us, with some extra assumptions, to apply our stability result.

Now remark that the previous construction of the normal and the natural maps is highly based on the fact that if $C(u)$ is closed and convex, then the set-valued mapping $\Pi(u, \cdot): z \rightrightarrows(N(C(u), \cdot)+I)^{-1}(z)$ is in fact single-valued (and equal to $P_{C(u)}(\cdot)$ ). Thus, to deal with nonconvex sets $C(u)$, it is sufficient to assume directly that $\Pi(u, \cdot)$ is single-valued. So, instead of considering at this step a general class (see Section 4) of nonconvex sets $C(u)$ for which $\Pi(u, \dot{)}$ is single-valued, the above comments lead us to the following first hypothesis.

Hypothesis 1. We suppose that there exist sets $U \subseteq \mathcal{U}$ and $X, Z \subseteq \mathcal{H}$ such that the localization of $\Pi$ on $U \times Z \times X$ defined by

$$
\begin{aligned}
& \Pi_{X}: \quad U \times Z \quad \rightrightarrows X \\
& (u, z) \quad \mapsto \quad(N(C(u), \cdot)+I)^{-1} \cap X
\end{aligned}
$$

is single-valued and we denote by $\pi_{X}$ its associated mapping, that is,

$$
\Pi_{X}(u, z)=\left\{\pi_{X}(u, z)\right\}, \quad \forall(u, z) \in U \times Z .
$$

This hypothesis naturally leads to a second one. Indeed, as it appears clearly in the natural map, putting

$$
\zeta(v, w, x):=w-\phi(v, x)+x,
$$

we have to take the image of $\zeta(v, w, x)$ under the mapping $\Pi(u, \cdot)$.

Therefore, restricting the single-valuedness of $\Pi(u, \cdot)$ to $Z$ requires to suppose that $\zeta(v, w, x) \in Z$ in order to make sure that $\Pi_{X}(u, \zeta(v, w, x))$ is a singleton set, and so the natural mapping is still well defined.

Consequently, we have to assume that, for all $(v, w, x) \in \mathcal{V} \times \mathcal{H} \times X$, the inclusion $\zeta(v, w, x) \in Z$ holds. So we restrict again the sets on which we make the assumptions. 
Hypothesis 2. Under notation of Hypothesis 1, we suppose that there exist sets $V \subseteq \mathcal{V}$ and $W \subseteq \mathcal{H}$ such that, for all $(v, w, x) \in V \times W \times X$, one has,

$$
\zeta(v, w, x) \in Z
$$

This assumption seems natural when dealing with the natural map but do not seem necessarily at first sight when considering the normal map.

At this point we can generalize the normal and natural mappings.

Definition 3.1. Let $U \subseteq \mathcal{U}, V \subseteq \mathcal{V}$ and $W, X, Z \subseteq \mathcal{H}$ satisfying Hypotheses 1 and 2 and define $\Pi_{X}$ and $\pi_{X}$ as in Hypothesis 1.

We define the local normal map by

$$
\begin{aligned}
f_{X}: U \times V \times Z & \rightarrow \mathcal{H} \\
(u, v, z) & \mapsto z-\pi_{X}(u, z)+\phi\left(v, \pi_{X}(u, z)\right)
\end{aligned}
$$

and the local natural map by

$$
\begin{aligned}
g_{X}: U \times V \times W \times X & \rightarrow \mathcal{H}, \\
(u, v, w, x) & \mapsto x-\pi_{X}(u, w-\phi(v, x)+x) .
\end{aligned}
$$

Finally, the third assumption below seems quite natural since we want to obtain an homeomorphism between the sets of solutions of the multi-valued problem and the single-valued ones, and then to apply the stability of section two.

Hypothesis 3. Under notation of Hypotheses 1 and 2, we assume that $\pi_{X}$ is continuous on $U \times Z$ and $\phi$ is continuous on $V \times X$.

It is worth noting here that those three hypotheses are not too strong in the sense that, as we will see in the last section, they are fulfilled by a general class of set-valued mappings called prox-regular under a certain type of continuity assumption.

We are now ready to show the links between the different solutions sets.

\subsection{Homeomorphism result}

So far we are facing three different problems, one multi-valued and two others single-valued. We first need to define the sets of solutions of those problems.

Definition 3.2. Let $U \subseteq \mathcal{U}, V \subseteq \mathcal{V}$ and $W, X, Z \subseteq \mathcal{H}$ satisfying Hypotheses 1,2 and 3, and define $\Pi_{X}$ and $\pi_{X}$ as in Hypothesis 1 .

We denote by $\mathcal{M}$ the set of solutions of $(\mathrm{VI}(\mathrm{u}, \mathrm{v}, \mathrm{w}))$ associated to $U, V, W$ and $X$, that is,

$$
\mathcal{M}:=\{(u, v, w, x) \in U \times V \times W \times X: w \in N(C(u), x)+\phi(v, x)\} .
$$

Likewise we denote by $\mathcal{N}$ the set of solutions of the local normal map equation associated to $U, V, W$ and $Z$, that is,

$$
\mathcal{N}:=\left\{(u, v, w, z) \in U \times V \times W \times Z: w=f_{X}(u, v, z)\right\},
$$

and by $\mathcal{L}$ the set of solutions of the local natural map equation associated to $U, V, W$ and $Z$, that is,

$$
\mathcal{L}:=\left\{(u, v, w, x) \in U \times V \times W \times X: 0=g_{X}(u, v, w, x)\right\} .
$$

It is worth noting here that the notations $\mathcal{M}, \mathcal{N}$ and $\mathcal{L}$ do not show the dependence in $U, V, W, X$ and $Z$, but we have to keep in mind that for different sets $U, V, W, X$ and $Z$ we get different $\mathcal{M}, \mathcal{N}$ and $\mathcal{L}$.

Now, let us start by showing the equality between $\mathcal{M}$ and $\mathcal{L}$.

Theorem 3.3. Let $U \subseteq \mathcal{U}, V \subseteq \mathcal{V}$ and $W, X, Z \subseteq \mathcal{H}$ satisfying Hypotheses 1, 2 and 3, and define $\Pi_{X}$ and $\pi_{X}$ as in Hypothesis 1 , and, $\mathcal{M}$ and $\mathcal{L}$ as in Definition 3.2.

Then one has

$$
\mathcal{M}=\mathcal{L}
$$


Proof. Let $(u, v, w, x)$ be in $\mathcal{M}$, then $w \in N(C(u), x)+\phi(v, x)$ with $(u, v, w, x) \in U \times V \times W \times X$. This gives

$$
w-\phi(v, x)+x \in N(C(u), x)+x,
$$

which is equivalent to

$$
x \in(N(C(u), \cdot)+I)^{-1}(w-\phi(v, x)+x) .
$$

Since $x \in X$, we have

$$
x \in(N(C(u), \cdot)+I)^{-1}(w-\phi(v, x)+x) \cap X=\Pi_{X}(u, w-\phi(v, x)+x) .
$$

Moreover $w-\phi(v, x)+x=\zeta(v, w, x)$ and by Hypothesis $2, \zeta(v, w, x) \in Z$ which implies that $(u, w-\phi(v, x)+x) \in$ $U \times Z$. Now using Hypothesis 1 , we have that $\Pi_{X}$ is single valued on $U \times Z$ with associated mapping $\pi_{X}$, so we obtain

$$
x=\pi_{X}(u, w-\phi(v, x)+x)
$$

i.e.,

$$
0=x-\pi_{X}(u, w-\phi(v, x)+x)=g_{X}(u, v, w, x),
$$

hence $(u, v, w, x) \in \mathcal{L}$.

Now assuming $(u, v, w, x) \in \mathcal{L}$ gives $(u, v, w, x) \in U \times V \times W \times X$ and

$$
0=x-\pi_{X}(u, w-\phi(v, x)+x),
$$

which implies by definition of $\pi_{X}$,

$$
x \in(N(C(u), \cdot)+I)^{-1}(w-\phi(v, x)+x),
$$

i.e.,

$$
w \in N(C(u), x)+\phi(v, x),
$$

hence $(u, v, w, x) \in \mathcal{M}$.

Now we establish Robinson's homeomorphism result to possibly infinite dimensional Hilbert spaces.

Theorem 3.4. Let $U \subseteq \mathcal{U}, V \subseteq \mathcal{V}$ and $X, Z \subseteq \mathcal{H}$ be as in Hypotheses 1, 2 and 3, and define $\pi_{X}$ as in Hypothesis 1, $f_{X}$ as in Definition 3.1, $\zeta$ as in (4), and $\mathcal{M}, \mathcal{N}$ and $\mathcal{L}$ as in Definition 3.2.

Then the map

$$
\begin{aligned}
\mu: \quad \mathcal{M} & \rightarrow \mathcal{N} \\
(u, v, w, x) & \mapsto(u, v, w, \zeta(v, w, x))
\end{aligned}
$$

is an homeomorphism from $\mathcal{M}$ to $\mathcal{N}$, and its inverse map is given by

$$
\begin{aligned}
\nu: \quad \mathcal{N} & \rightarrow \mathcal{M} \\
(u, v, w, z) & \mapsto\left(u, v, w, \pi_{X}(u, z)\right) .
\end{aligned}
$$

Proof. First of all, it is not difficult to see that, under the assumptions, $\mu$ and $\nu$ are continuous on their domain.

Now we show that $\mu$ takes its values in $\mathcal{N}$. Let $(u, v, w, x) \in \mathcal{M}$. We have $(u, v, w, x) \in U \times V \times W \times X$, and $\mu(u, v, w, x)=(u, v, w, \zeta(v, w, x))$. Set

$$
z:=\zeta(v, w, x)=w-\phi(v, x)+x .
$$

Thus $z \in Z$ by Hypothesis 2 . We have to show that $f_{X}(u, v, z)=w$, that is,

$$
\phi\left(v, \pi_{X}(u, z)\right)+z-\pi_{X}(u, z)=w .
$$

As $(u, v, w, x) \in \mathcal{M}$, we have by Theorem 3.3 that $(u, v, w, x) \in \mathcal{L}$, i.e., $x=\pi_{X}(u, z)$. Thus replacing $x$ by $\pi_{X}(u, z)$ in (5) gives

$$
z=w-\phi\left(v, \pi_{X}(u, z)\right)+\pi_{X}(u, z),
$$

or equivalently,

$$
w=\phi\left(v, \pi_{X}(u, z)\right)+z-\pi_{X}(u, z)=f_{X}(u, v, z)
$$


which shows that $\mu(u, v, w, x) \in \mathcal{N}$.

We claim that $(\nu \circ \mu)(u, v, w, x)=(u, v, w, x)$. Indeed

$$
(\nu \circ \mu)(u, v, w, x)=\nu(u, v, w, z)=\left(u, v, w, \pi_{X}(u, z)\right)=(u, v, w, x)
$$

since we showed above that $x=\pi_{X}(u, z)$. So we have $\nu \circ \mu=I_{\mathcal{M}}$.

Now we show that $\nu$ takes its values in $\mathcal{M}$. Let $(u, v, w, z) \in \mathcal{N}$. We have $(u, v, w, z) \in U \times V \times W \times Z$ and $\nu(u, v, w, z)=\left(u, v, w, \pi_{X}(u, z)\right)$. Set $x:=\pi_{X}(u, z)$ and note by definition of $\pi_{X}$ that $x \in X$.

We know that $f_{X}(u, v, z)=w$, i.e.,

$$
\phi\left(v, \pi_{X}(u, z)\right)+z-\pi_{X}(u, z)=w,
$$

or equivalently,

$$
z=w-\phi(v, x)+x=\zeta(v, w, x)
$$

since $x=\pi_{X}(u, z)$. Therefore, we can write $x=\pi_{X}(u, \zeta(v, w, x))$, or equivalently, $0=x-\pi_{X}(u, \zeta(v, w, x))$ which gives $g_{X}(u, v, w, x)=0$. This implies that $(u, v, w, x) \in \mathcal{L}$, and so, by Theorem $3.3,(u, v, w, x) \in \mathcal{M}$, i.e., $\nu(u, v, w, z) \in \mathcal{M}$ as desired.

Finally we deduce from (6) that

$$
(\mu \circ \nu)(u, v, w, z)=\mu(u, v, w, x)=(u, v, w, \zeta(v, w, x))=(u, v, w, z),
$$

which shows that $\mu \circ \nu=I_{\mathcal{N}}$ and concludes the proof.

We now see how this result allows us to solve two stability problems of solutions of $(\mathrm{VI}(\mathrm{u}, \mathrm{v}, \mathrm{w}))$.

\subsection{Stability of solutions of variational inequalities}

In this section, we study two stability results of solutions of $(\mathrm{VI}(\mathrm{u}, \mathrm{v}, \mathrm{w}))$.

Assuming $\left(u_{0}, v_{0}, w_{0}, x_{0}\right) \in \mathcal{U} \times \mathcal{V} \times \mathcal{H}^{2}$ and

$$
w_{0} \in N\left(C\left(u_{0}\right), x_{0}\right)+\phi\left(v_{0}, x_{0}\right),
$$

we first study how the solution $x$ evolves with a variation of $w$, i.e., the stability with respect to $w$ of the solution $x$ of the inequality

$$
w \in N\left(C\left(u_{0}\right), x\right)+\phi\left(v_{0}, x\right) .
$$

In the second problem, $w_{0}$ will be the fixed variable, and the variation will be with respect to $(u, v)$, that is, we will examine the behavior (with respect to $(u, v)$ ) of the solution $x$ of the variational inequality

$$
w_{0} \in N(C(u), x)+\phi(v, x) .
$$

Let us introduce some notation in order to solve (Prob1). Let $u_{0} \in \mathcal{U}, v_{0} \in \mathcal{V}$ and $W, X, Z \subseteq \mathcal{H}$ be such that $U:=\left\{u_{0}\right\}, V:=\left\{v_{0}\right\}, W, X$ and $Z$ satisfy Hypotheses 1,2 and 3. We can then consider $\Pi_{X}, \pi_{X}$ and $\mathcal{M}, \mathcal{N}$ as above.

However, to emphasize the dependence in $\left(u_{0}, v_{0}\right)$, we will denote $\mathcal{M}$, resp. $\mathcal{N}$, by $\mathcal{M}\left(u_{0}, v_{0}\right)$, resp. $\mathcal{N}\left(u_{0}, v_{0}\right)$. Then we construct the sets $\mathcal{X}$, resp. $\mathcal{W}$, as the projection of $\mathcal{M}\left(u_{0}, v_{0}\right)$ on $X$, resp. on $W$, and $\mathcal{Z}$ as the projection of $\mathcal{N}\left(u_{0}, v_{0}\right)$ on $Z$, which means,

$$
\begin{gathered}
\mathcal{X}=\left\{x \in X: \exists w \in W \text { s.t. } w \in N\left(C\left(u_{0}\right), x\right)+\phi\left(v_{0}, x\right) \text { and } \zeta\left(v_{0}, w, x\right) \in Z\right\}, \\
\mathcal{W}=\left\{w \in W: \exists x \in X \text { s.t. } w \in N\left(C\left(u_{0}\right), x\right)+\phi\left(v_{0}, x\right) \text { and } \zeta\left(v_{0}, w, x\right) \in Z\right\}, \\
\mathcal{Z}=\left\{z \in Z: \exists x \in X, \exists w \in W \text { s.t. } w \in N\left(C\left(u_{0}\right), x\right)+\phi\left(v_{0}, x\right) \text { and } z=\zeta\left(v_{0}, w, x\right)\right\} .
\end{gathered}
$$

Finally, as we want to study how $x$ evolves under a variation of $w$, we naturally define the set-valued mapping

$$
\begin{aligned}
M\left(u_{0}, v_{0}\right): \mathcal{W} & \rightrightarrows \mathcal{X} . \\
w & \mapsto\left\{x \in X:\left(u_{0}, v_{0}, w, x\right) \in \mathcal{M}\left(u_{0}, v_{0}\right)\right\}
\end{aligned}
$$


Theorem 3.5. Let $u_{0} \in \mathcal{U}, v_{0} \in \mathcal{V}$ and $W, X, Z \subseteq \mathcal{H}$ be such that $U:=\left\{u_{0}\right\}, V:=\left\{v_{0}\right\}, W, X$ and $Z$ satisfy Hypotheses 1, 2 and 3 and define $\mathcal{M}\left(u_{0}, v_{0}\right), \mathcal{N}\left(u_{0}, v_{0}\right), \mathcal{X}, \mathcal{W}, \mathcal{Z}$ and $M\left(u_{0}, v_{0}\right)$ as above.

Then the map $f_{X}\left(u_{0}, v_{0}, \cdot\right): \mathcal{Z} \rightarrow \mathcal{W}$ is an homeomorphism if and only if the set-valued mapping $M\left(u_{0}, v_{0}\right)$ is single-valued on $\mathcal{W}$ and its associated mapping $m$ is continuous on $\mathcal{W}$.

In this case, one has for all $w \in \mathcal{W}$,

$$
m(w)=\pi_{X}\left(u_{0}, f_{X}\left(u_{0}, v_{0}, \cdot\right)^{-1}(w)\right)
$$

and

$$
f_{X}\left(u_{0}, v_{0}, \cdot\right)^{-1}(w)=\zeta\left(u_{0}, v_{0}, m(w)\right)
$$

Further

$$
\mathcal{M}\left(u_{0}, v_{0}\right)=\left\{\left(u_{0}, v_{0}\right)\right\} \times\{(w, m(w)): w \in \mathcal{W}\}
$$

and

$$
\mathcal{N}\left(u_{0}, v_{0}\right)=\left\{\left(u_{0}, v_{0}\right)\right\} \times\left\{\left(w, f_{X}\left(u_{0}, v_{0}, \cdot\right)^{-1}(w)\right): w \in \mathcal{W}\right\}
$$

Proof. First we show that $f_{X}\left(u_{0}, v_{0}, \cdot\right)$ takes its values in $\mathcal{W}$. Let $z \in \mathcal{Z}$. There exists some $w \in W$ such that $\left(u_{0}, v_{0}, w, z\right) \in \mathcal{N}\left(u_{0}, v_{0}\right)$, which implies that $w=f_{X}\left(u_{0}, v_{0}, z\right)$ by the definition of $\mathcal{N}\left(u_{0}, v_{0}\right)$, so

$$
\left(u_{0}, v_{0}, f_{X}\left(u_{0}, v_{0}, z\right), z\right) \in \mathcal{N}\left(u_{0}, v_{0}\right) .
$$

Therefore, we have by Theorem 3.4 that

$$
\nu\left(u_{0}, v_{0}, f_{X}\left(u_{0}, v_{0}, z\right), z\right)=\left(u_{0}, v_{0}, f_{X}\left(u_{0}, v_{0}, z\right), \pi_{X}\left(u_{0}, z\right)\right) \in \mathcal{M}\left(u_{0}, v_{0}\right)
$$

which implies that $f_{X}\left(u_{0}, v_{0}, z\right) \in \mathcal{W}$ by the definition of $\mathcal{W}$.

$\Rightarrow$ : Let us suppose that $f_{X}\left(u_{0}, v_{0}, \cdot\right)$ is an homeomorphism from $\mathcal{Z}$ to $\mathcal{W}$ and let us show that $M\left(u_{0}, v_{0}\right)$ is single-valued on $\mathcal{W}$ with its associated mapping $m$ continuous on $\mathcal{W}$.

First we show that $\operatorname{dom} M\left(u_{0}, v_{0}\right)=\mathcal{W}$. Let $w \in \mathcal{W}$. There exists $x \in X$ such that $\left(u_{0}, v_{0}, w, x\right) \in$ $\mathcal{M}\left(u_{0}, v_{0}\right)$, i.e., $x \in M\left(u_{0}, v_{0}\right)(w)$, and so, $w \in \operatorname{dom} M\left(u_{0}, v_{0}\right)$, which leads to $\operatorname{dom} M\left(u_{0}, v_{0}\right)=\mathcal{W}$.

Now we show that, for any $w \in \mathcal{W}$ and any $x_{1}, x_{2} \in M\left(u_{0}, v_{0}\right)(w)$, we have that $x_{1}=x_{2}$. So let $w \in \mathcal{W}$ and $x_{1}, x_{2} \in M\left(u_{0}, v_{0}\right)(w)$. For $i=1,2$, set $z_{i}:=\zeta\left(v_{0}, w, x_{i}\right)$. We have by definition of $M\left(u_{0}, v_{0}\right)$ that $\left(u_{0}, v_{0}, w, x_{i}\right) \in \mathcal{M}\left(u_{0}, v_{0}\right)$, so we deduce by Theorem 3.4 that

$$
\left(u_{0}, v_{0}, w, z_{i}\right)=\mu\left(u_{0}, v_{0}, w, x_{i}\right) \in \mathcal{N}\left(u_{0}, v_{0}\right)
$$

i.e., $f_{X}\left(u_{0}, v_{0}, z_{i}\right)=w$, which gives that $f_{X}\left(u_{0}, v_{0}, z_{1}\right)=f_{X}\left(u_{0}, v_{0}, z_{2}\right)$. We then use the fact that $f_{X}\left(u_{0}, v_{0}, \cdot\right)$ is one-to-one (as an homeomorphism) to obtain that $z_{1}=z_{2}$. Therefore, using Theorem 3.4 once again, we conclude that

$$
\begin{aligned}
\left(u_{0}, v_{0}, w, x_{1}\right) & =(\nu \circ \mu)\left(u_{0}, v_{0}, w, x_{1}\right) \\
& =\nu\left(u_{0}, v_{0}, w, z_{1}\right) \\
& =\nu\left(u_{0}, v_{0}, w, z_{2}\right) \\
& =(\nu \circ \mu)\left(u_{0}, v_{0}, w, x_{2}\right) \\
& =\left(u_{0}, v_{0}, w, x_{2}\right)
\end{aligned}
$$

hence $x_{1}=x_{2}$.

Thus, we have shown that $M\left(u_{0}, v_{0}\right)$ is single-valued on $\mathcal{W}$. Let us call $m$ its associated mapping. We claim that, $m(w)=\pi_{X}\left(u_{0}, f_{X}\left(u_{0}, v_{0}, \cdot\right)^{-1}(w)\right)$, for all $w \in \mathcal{W}$. To do so, we prove that, for every $w \in \mathcal{W}$,

$$
\pi_{X}\left(u_{0}, f_{X}\left(u_{0}, v_{0}, \cdot\right)^{-1}(w)\right) \in M\left(u_{0}, v_{0}\right)(w) .
$$

Let $w \in \mathcal{W}$ and set $z=f_{X}\left(u_{0}, v_{0}, \cdot\right)^{-1}(w)$ which implies that $z \in \mathcal{Z}$. Set also $x=\pi_{X}\left(u_{0}, z\right)$ and show that $x \in M\left(u_{0}, v_{0}\right)(w)$. By the definition of $z$, we have that $f_{X}\left(u_{0}, v_{0}, z\right)=w$ with $z \in \mathcal{Z} \subseteq Z$, which gives that $\left(u_{0}, v_{0}, w, z\right) \in \mathcal{N}\left(u_{0}, v_{0}\right)$. So from Theorem 3.4 we deduce that

$$
\left(u_{0}, v_{0}, w, x\right)=\nu\left(u_{0}, v_{0}, w, z\right) \in \mathcal{M}\left(u_{0}, v_{0}\right)
$$


so $x \in M\left(u_{0}, v_{0}\right)(w)$ which proves the claim.

Now, since $m(w)=\pi_{X}\left(u_{0}, f_{X}\left(u_{0}, v_{0}, \cdot\right)^{-1}(w)\right)$, for all $w \in \mathcal{W}$, it is not hard to see that, under the homeomorphism hypothesis on $f_{X}\left(u_{0}, v_{0}, \cdot\right)$, the mapping $m$ is continuous on $\mathcal{W}$ as a composite of continuous mappings, which concludes the proof of the first implication.

$\Leftarrow$ : Let us suppose that $M\left(u_{0}, v_{0}\right)$ is single-valued on $\mathcal{W}$ and that its associated mapping $m$ is continuous on $\mathcal{W}$, and let us show that $f_{X}\left(u_{0}, v_{0}, \cdot\right)$ is an homeomorphism from $\mathcal{Z}$ to $\mathcal{W}$ with $f_{X}\left(u_{0}, v_{0}, \cdot\right)^{-1}(w)=$ $\zeta\left(v_{0}, w, m_{X}(w)\right)$, for all $w \in \mathcal{W}$. Consider the mapping

$$
\begin{aligned}
\varrho: \mathcal{W} & \rightarrow \mathcal{Z} . \\
w & \mapsto \zeta\left(v_{0}, w, m(w)\right)
\end{aligned}
$$

Once again, it is not hard (using Theorem 3.4) to see that, on one hand, $\varrho$ takes its values in $\mathcal{Z}$ and that, on the other hand, the mappings $f_{X}\left(u_{0}, v_{0}, \cdot\right)$ and $\varrho$ are continuous on $\mathcal{Z}$ and $\mathcal{W}$ respectively. We show that

$$
f_{X}\left(u_{0}, v_{0}, \cdot\right) \circ \varrho=I_{\mathcal{W}} \quad \text { and } \quad \varrho \circ f_{X}\left(u_{0}, v_{0}, \cdot\right)=I_{\mathcal{Z}} .
$$

Let $w \in \mathcal{W}$. We have by definition of $m$ that $\left(u_{0}, v_{0}, w, m(w)\right) \in \mathcal{M}\left(u_{0}, v_{0}\right)$. So, applying Theorem 3.4 gives that

$$
\left(u_{0}, v_{0}, w, \varrho(w)\right)=\left(u_{0}, v_{0}, w, \zeta\left(v_{0}, w, m(w)\right)\right)=\mu\left(u_{0}, v_{0}, w, m(w)\right) \in \mathcal{N}\left(u_{0}, v_{0}\right),
$$

which gives, by definition of $\mathcal{N}\left(u_{0}, v_{0}\right)$, that $w=f_{X}\left(u_{0}, v_{0}, m(w)\right)$, that is, $\left(f_{X}\left(u_{0}, v_{0}, \cdot\right) \circ m\right)(w)=w$.

Now let $z \in \mathcal{Z}$. Set $w:=f_{X}\left(u_{0}, v_{0}, z\right)$. Then, by the definition of $\mathcal{N}\left(u_{0}, v_{0}\right)$ we have that $\left(u_{0}, v_{0}, w, z\right) \in$ $\mathcal{N}\left(u_{0}, v_{0}\right)$, and so, by Theorem 3.4

$$
\left(u_{0}, v_{0}, w, z\right)=(\mu \circ \nu)\left(u_{0}, v_{0}, w, z\right)=\left(u_{0}, v_{0}, w, \zeta\left(v_{0}, w, \pi_{X}\left(u_{0}, z\right)\right)\right)
$$

hence $z=\zeta\left(v_{0}, w, \pi_{X}\left(u_{0}, z\right)\right)$. Moreover, $\left(u_{0}, v_{0}, w, z\right) \in \mathcal{N}\left(u_{0}, v_{0}\right)$ implies, by Theorem 3.4, that

$$
\nu\left(u_{0}, v_{0}, w, z\right)=\left(u_{0}, v_{0}, w, \pi_{X}\left(u_{0}, z\right)\right) \in \mathcal{M}\left(u_{0}, v_{0}\right)
$$

so $\pi_{X}\left(u_{0}, z\right) \in M\left(u_{0}, v_{0}\right)(w)$, which gives that $\pi_{X}\left(u_{0}, z\right)=m(w)$. We deduce that

$$
z=\zeta\left(v_{0}, w, m(w)\right)=\varrho(w)=\left(\varrho \circ f_{X}\left(u_{0}, v_{0}, \cdot\right)\right)(z)
$$

which concludes the equivalence.

We finish the proof by showing that, through the above equivalence, we have

$$
\mathcal{M}\left(u_{0}, v_{0}\right)=\left\{\left(u_{0}, v_{0}\right)\right\} \times\{(w, m(w)): w \in \mathcal{W}\}
$$

and

$$
\mathcal{N}\left(u_{0}, v_{0}\right)=\left\{\left(u_{0}, v_{0}\right)\right\} \times\left\{\left(w, f_{X}\left(u_{0}, v_{0}, \cdot\right)^{-1}(w)\right): w \in \mathcal{W}\right\} .
$$

Let $\left(u_{0}, v_{0}, w_{0}, x_{0}\right) \in \mathcal{M}\left(u_{0}, v_{0}\right)$. So $w_{0} \in \mathcal{W}$ by definition of $\mathcal{W}$ and, by the definition of $M\left(u_{0}, v_{0}\right)$ we have that $x_{0} \in M\left(u_{0}, v_{0}\right)\left(w_{0}\right)$. But as $M\left(u_{0}, v_{0}\right)$ is single-valued and $m(\cdot)$ is its associated mapping, we obtain $x_{0}=m\left(w_{0}\right)$, hence $\left(u_{0}, v_{0}, w_{0}, x_{0}\right)=\left(u_{0}, v_{0}, w_{0}, m\left(w_{0}\right)\right)$ with $w_{0} \in \mathcal{W}$, so

$$
\mathcal{M}\left(u_{0}, v_{0}\right) \subseteq\left\{\left(u_{0}, v_{0}\right)\right\} \times\{(w, m(w)): w \in \mathcal{W}\} .
$$

If now $\left(u_{0}, v_{0}, w_{0}, x_{0}\right) \in\left\{\left(u_{0}, v_{0}\right)\right\} \times\{(w, m(w)): w \in \mathcal{W}\}$. We have $x_{0}=m\left(w_{0}\right)$, i.e., $x_{0} \in M\left(u_{0}, v_{0}\right)\left(w_{0}\right)$, which gives $\left(u_{0}, v_{0}, w_{0}, x_{0}\right) \in \mathcal{M}\left(u_{0}, v_{0}\right)$ and so

$$
\left\{\left(u_{0}, v_{0}\right)\right\} \times\{(w, m(w)): w \in \mathcal{W}\} \subseteq \mathcal{M}\left(u_{0}, v_{0}\right) .
$$

To show that

$$
\mathcal{N}\left(u_{0}, v_{0}\right)=\left\{\left(u_{0}, v_{0}\right)\right\} \times\left\{\left(w, f_{X}\left(u_{0}, v_{0}, \cdot\right)^{-1}(w)\right): w \in \mathcal{W}\right\}
$$

it suffices to observe that if $\left(u_{0}, v_{0}, w_{0}, z_{0}\right) \in \mathcal{N}\left(u_{0}, v_{0}\right)$, we have by Theorem 3.4 that

$$
\left(u_{0}, v_{0}, w_{0}, z_{0}\right)=(\mu \circ \nu)\left(u_{0}, v_{0}, w_{0}, z_{0}\right)
$$


with $\nu\left(u_{0}, v_{0}, w_{0}, z_{0}\right) \in \mathcal{M}\left(u_{0}, v_{0}\right)$. But as

$$
\mathcal{M}\left(u_{0}, v_{0}\right)=\left\{\left(u_{0}, v_{0}\right)\right\} \times\{(w, m(w)): w \in \mathcal{W}\}
$$

we have

$$
\left(u_{0}, v_{0}, w_{0}, z_{0}\right)=\mu\left(u_{0}, v_{0}, w_{0}, m\left(w_{0}\right)\right)=\left(u_{0}, v_{0}, w_{0}, \zeta\left(u_{0}, v_{0}, m\left(w_{0}\right)\right)\right)
$$

by definition of $\mu$. But we saw that $\zeta\left(u_{0}, v_{0}, m\left(w_{0}\right)\right)=f_{X}\left(u_{0}, v_{0}, \cdot\right)^{-1}\left(w_{0}\right)$, hence

$$
\left(u_{0}, v_{0}, w_{0}, z_{0}\right)=\left(u_{0}, v_{0}, w_{0}, f_{X}\left(u_{0}, v_{0}, \cdot\right)^{-1}\left(w_{0}\right)\right)
$$

which proves

$$
\mathcal{N}\left(u_{0}, v_{0}\right) \subseteq\left\{\left(u_{0}, v_{0}\right)\right\} \times\left\{\left(w, f_{X}\left(u_{0}, v_{0}, \cdot\right)^{-1}(w)\right): w \in \mathcal{W}\right\}
$$

We apply the same arguments to show the reverse inclusion and conclude the proof.

Now we study Problem (Prob2) to see how the solution $x$ evolves in function of $(u, v)$ when $w_{0}$ is fixed. To do so, we first remark that, for fixed $\left(u_{0}, v_{0}, w_{0}\right)$, the natural map $g_{X}\left(u_{0}, v_{0}, w_{0}, \cdot\right)$, and the normal map $f_{X}\left(u_{0}, v_{0}, \cdot\right)$, are both perturbations of the identity.

This will allow us to apply the stability result obtained in Section 2, to get stability results for the equations

$$
0=g_{X}\left(u, v, w_{0}, x\right)
$$

and

$$
w_{0}=f_{X}(u, v, x)
$$

Since $\mathcal{M}=\mathcal{L}$, stability result for (7) directly yields stability for Problem (Prob2), but when considering the normal map, we will need to link solutions of (8) to solutions of Problem (Prob2). This is the purpose of the following lemma. But first, we need once again to introduce notation.

Let $w_{0} \in \mathcal{H}, U \subseteq \mathcal{U}, V \subseteq \mathcal{V}$ and $X, Z \subseteq \mathcal{H}$ such that $U, V, W:=\left\{w_{0}\right\}, X$ and $Z$ satisfy Hypotheses 1,2 and 3 .

As we did previously for $\mathcal{M}\left(u_{0}, v_{0}\right)$ and $\mathcal{N}\left(u_{0}, v_{0}\right)$, we will denote by $\mathcal{M}\left(w_{0}\right)$, resp. $\mathcal{N}\left(w_{0}\right)$ and $\mathcal{L}\left(w_{0}\right)$, the sets $\mathcal{M}$, resp. $\mathcal{N}$ and $\mathcal{L}$ with $W:=\left\{w_{0}\right\}$, to emphasize the fact that $w_{0}$ is fixed.

From there, we define the set-valued mappings

$$
\begin{aligned}
M\left(w_{0}\right): U \times V & \rightrightarrows X \\
(u, v) & \mapsto\left\{x \in X:\left(u, v, w_{0}, x\right) \in \mathcal{M}\left(w_{0}\right)\right\}
\end{aligned}
$$

and

$$
\begin{aligned}
N\left(w_{0}\right): U \times V & \rightrightarrows Z . \\
(u, v) & \mapsto\left\{z \in Z:\left(u, v, w_{0}, z\right) \in \mathcal{N}\left(w_{0}\right)\right\}
\end{aligned}
$$

We can now give the lemma showing that those two set-valued mappings behave similarly on a continuity point of view.

Lemma 3.6. Let $w_{0} \in \mathcal{H}, U \subseteq \mathcal{U}, V \subseteq \mathcal{V}$ and $X, Z \subseteq \mathcal{H}$ such that $U, V, W:=\left\{w_{0}\right\}, X$ and $Z$ satisfy Hypotheses 1, 2 and 3, and define $\mathcal{M}\left(w_{0}\right), \mathcal{N}\left(w_{0}\right), M\left(w_{0}\right)$ and $N\left(w_{0}\right)$ as above.

Considering $\left(u_{0}, v_{0}\right) \in U \times V$, we have that $M\left(w_{0}\right)$ is lower semicontinuous at $\left(u_{0}, v_{0}\right)$ if and only if $N\left(w_{0}\right)$ is lower semicontinuous (lsc for short) at $\left(u_{0}, v_{0}\right)$.

Proof. Let us suppose that $M\left(w_{0}\right)$ is lsc at $\left(u_{0}, v_{0}\right)$ and show $N\left(w_{0}\right)$ is lsc at $\left(u_{0}, v_{0}\right)$ too. Let $O_{Z}$ be an open subset of $Z$ for the topology induced by the one of $\mathcal{H}$ such that $N\left(w_{0}\right)\left(u_{0}, v_{0}\right) \cap O_{Z} \neq \emptyset$ (if such an open set does not exist then $N\left(w_{0}\right)$ is trivially lsc at $\left.\left(u_{0}, v_{0}\right)\right)$. So by definition of the induced topology, there exists an open subset $O_{\mathcal{H}}$ of $\mathcal{H}$ such that $O_{Z}=Z \cap O_{\mathcal{H}}$, hence $N\left(w_{0}\right)\left(u_{0}, v_{0}\right) \cap Z \cap O_{\mathcal{H}} \neq \emptyset$. Therefore, there exists $z \in O_{\mathcal{H}}$ such that $\left(u_{0}, v_{0}, w_{0}, z\right) \in \mathcal{N}\left(w_{0}\right)$ which gives by Theorem 3.4 that

$$
\left(u_{0}, v_{0}, w_{0}, z\right)=(\mu \circ \nu)\left(u_{0}, v_{0}, w_{0}, z\right)=\left(u_{0}, v_{0}, w_{0}, \zeta\left(v_{0}, w_{0}, \pi_{X}\left(u_{0}, z\right)\right)\right)
$$

so $z=\zeta\left(v_{0}, w_{0}, \pi_{X}\left(u_{0}, z\right)\right)$. Now using the continuity of $\zeta\left(\cdot, w_{0}, \cdot\right)$ we have that $\zeta\left(\cdot, w_{0}, \cdot\right)^{-1}\left(O_{\mathcal{H}}\right)$ is a neighbourhood of $\left(v_{0}, \pi_{X}\left(u_{0}, z\right)\right)$ for the product topology on $\mathcal{V} \times \mathcal{H}$. Thus, there exists a neighbourhood $V_{0}$ of $v_{0}$ for the topology on $\mathcal{V}$ and a neighbourhood $X_{0}$ of $\pi_{X}\left(u_{0}, z\right)$ for the topology on $\mathcal{H}$ such that 
$V_{0} \times X_{0} \subseteq \zeta\left(\cdot, w_{0}, \cdot\right)^{-1}\left(O_{\mathcal{H}}\right)$. Moreover by Theorem 3.4 we have also $\left(u_{0}, v_{0}, w_{0}, \pi_{X}\left(u_{0}, z\right)\right) \in \mathcal{M}\left(w_{0}\right)$, so $\pi_{X}\left(u_{0}, z\right) \in M\left(w_{0}\right)\left(u_{0}, v_{0}\right) \cap X_{0} \cap X$ which gives $M\left(w_{0}\right)\left(u_{0}, v_{0}\right) \cap O_{X} \neq \emptyset$ for the set $O_{X}=X_{0} \cap X$ which is open with respect to the topology on $X$ induced by $\mathcal{H}$.

We now use the fact that $M\left(w_{0}\right)$ is lsc at $\left(u_{0}, v_{0}\right)$ to obtain the existence of a neighbourhood $U_{0}$ of $u_{0}$ (for the topology on $U$ induced by the one of $\mathcal{U}$ ) and a neighbourhood $V_{1}$ of $v_{0}$ (for the topology on $V$ induced by the one of $\mathcal{V})$ such that, $M\left(w_{0}\right)(u, v) \cap O_{X} \neq \emptyset$, for all $(u, v) \in U_{0} \times V_{1}$. So setting $V_{2}=V_{0} \cap V_{1}$ which is still a neighbourhood of $v_{0}$ for the topology on $V$, fixing $(u, v) \in U_{0} \times V_{2}$ we can choose some $x \in M\left(w_{0}\right)(u, v) \cap X_{0}$.

Now by definition of $M\left(w_{0}\right)(u, v)$ we have $\left(u, v, w_{0}, x\right) \in \mathcal{M}\left(w_{0}\right)$ which gives by Theorem 3.4 that $\left(u, v, w_{0}, \zeta\left(v, w_{0}, x\right)\right) \in$ $\mathcal{N}\left(w_{0}\right)$, that is, $\zeta\left(v, w_{0}, x\right) \in N\left(w_{0}\right)(u, v)$. Also, using the definitions of $V_{0}$ and $X_{0}$ we have that $\zeta\left(v, w_{0}, x\right) \in$ $O_{\mathcal{H}}$, thus $\zeta\left(v, w_{0}, x\right) \in N\left(w_{0}\right)(u, v) \cap O_{\mathcal{H}}$. Consequently, for all $(u, v) \in U_{0} \times V_{2}$, we have

$$
N\left(w_{0}\right)(u, v) \cap O_{Z}=N\left(w_{0}\right)(u, v) \cap Z \cap O_{\mathcal{H}}=N\left(w_{0}\right)(u, v) \cap O_{\mathcal{H}} \neq \emptyset,
$$

hence $N\left(w_{0}\right)$ is lsc at $\left(u_{0}, v_{0}\right)$.

Let us suppose now $N\left(w_{0}\right)$ is lsc at $\left(u_{0}, v_{0}\right)$ and show that $M\left(w_{0}\right)$ is lsc at this point too.

Let $O_{X}$ be an open set of $X$ (for the topology induced by the one of $\mathcal{H}$ ) such that $M\left(w_{0}\right)\left(u_{0}, v_{0}\right) \cap O_{X} \neq \emptyset$. By definition of the induced topology, there exists an open set $O_{\mathcal{H}}$ of $\mathcal{H}$ such that $O_{X}=O_{\mathcal{H}} \cap X$. Now, because $\pi_{X}$ is defined and continuous on $U \times Z$ for the topology induced by the one of $\mathcal{U} \times \mathcal{H}$, the set $\pi_{X}^{-1}\left(O_{\mathcal{H}}\right)$ is open in $U \times Z$. So there exists an open set $O_{\mathcal{U} \times \mathcal{H}}$ of $\mathcal{U} \times \mathcal{H}$ such that $\pi_{X}^{-1}\left(O_{\mathcal{H}}\right)=(U \times Z) \cap O_{\mathcal{U} \times \mathcal{H}}$. Moreover, as $M\left(w_{0}\right)\left(u_{0}, v_{0}\right) \cap O_{\mathcal{H}} \neq \emptyset$, there exists $x \in O_{\mathcal{H}}$ such that $\left(u_{0}, v_{0}, w_{0}, x\right) \in \mathcal{M}\left(w_{0}\right)$, hence by

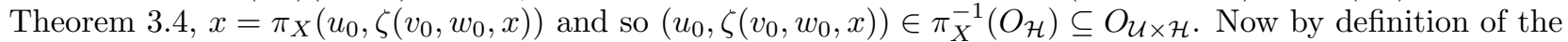
product topology, there exists a neighbourhood $U_{0}$ of $u_{0}$ in $\mathcal{U}$ and a neighbourhood $O_{\mathcal{H}}^{\prime}$ of $\zeta\left(v_{0}, w_{0}, x\right)$ in $\mathcal{H}$ such that $U_{0} \times O_{\mathcal{H}}^{\prime} \subseteq O_{\mathcal{U} \times \mathcal{H}}$. But by Theorem 3.4, we have $\left(u_{0}, v_{0}, w_{0}, \zeta\left(v_{0}, w_{0}, x\right)\right) \in \mathcal{N}\left(w_{0}\right)$ which implies that $\zeta\left(v_{0}, w_{0}, x\right) \in N\left(w_{0}\right)\left(u_{0}, v_{0}\right) \subseteq Z$, and by construction of $O_{\mathcal{H}}^{\prime}, \zeta\left(v_{0}, w_{0}, x\right) \in O_{\mathcal{H}}^{\prime}$. Therefore, considering the set $O_{Z}:=Z \cap O_{\mathcal{H}}^{\prime}$ which is open in $Z$ for the induced topology, we have $\zeta\left(v_{0}, w_{0}, x\right) \in N\left(w_{0}\right)\left(u_{0}, v_{0}\right) \cap O_{Z}$, hence $N\left(w_{0}\right)\left(u_{0}, v_{0}\right) \cap O_{Z} \neq \emptyset$.

Now, as $N\left(w_{0}\right)$ is lsc at $\left(u_{0}, v_{0}\right)$, there exists a neighbourhood $U_{1}$ of $u_{0}$ for the topology on $U$ induced by the one of $\mathcal{U}$ and a neighbourhood $V_{0}$ of $v_{0}$ for the topology on $V$ induced by the one of $\mathcal{V}$ such that, $N\left(w_{0}\right)(u, v) \cap O_{Z} \neq \emptyset$, for all $(u, v) \in U_{1} \times V_{0}$. So for any $(u, v) \in U_{1} \times V_{0}$, there exists $z \in O_{Z}$ such that $\left(u, v, w_{0}, z\right) \in \mathcal{N}\left(w_{0}\right)$, which implies that for every $(u, v) \in\left(U_{0} \cap U_{1}\right) \times V_{0}$, there exists $z \in O_{Z}$ such that $\left(u, v, w_{0}, \pi_{X}(u, z)\right) \in \mathcal{M}\left(w_{0}\right)$ according to Theorem 3.4, that is, $\pi_{X}(u, z) \in M\left(w_{0}\right)(u, v) \subseteq X$ with $\pi_{X}(u, z) \in O_{\mathcal{H}}$, hence $M\left(w_{0}\right)(u, v) \cap O_{X} \neq \emptyset$ and $M\left(w_{0}\right)$ is lsc at $\left(u_{0}, v_{0}\right)$, which concludes the proof.

We can now give Kien and Yao's stability result and Robinson's one in the Hilbert setting. The first result (that is, Theorem 3.7) corresponds, in the context of Hilbert space, to Theorem 3.1 in KY. In the rest of the paper, int $X$ denotes the topological interior of $X$.

Theorem 3.7. Let $u_{0} \in \mathcal{U}, v_{0} \in \mathcal{V}, w_{0}, x_{0} \in \mathcal{H}$ such that

$$
w_{0} \in N\left(C\left(u_{0}\right), x_{0}\right)+\phi\left(v_{0}, x_{0}\right) .
$$

Assume that there exist $U$, resp. $V, X$ and $Z$, neighbourhoods of $u_{0}$, resp. $v_{0}, x_{0}$ and $z_{0}:=\zeta\left(v_{0}, w_{0}, x_{0}\right)$ such that $U, V, W:=\left\{w_{0}\right\}, X$ and $Z$ satisfy Hypotheses 1, 2 and 3. Let $\mathcal{M}\left(w_{0}\right), \mathcal{N}\left(w_{0}\right)$ and $\mathcal{L}\left(w_{0}\right)$, and, $M\left(w_{0}\right)$ and $N\left(w_{0}\right)$ be as above.

Suppose also that:

(i) $X$ is closed and bounded;

(ii)

$$
\begin{aligned}
& \pi_{X}\left(\cdot, \zeta\left(\cdot, w_{0}, \cdot\right)\right): \quad U \times V \times X \quad \rightarrow \quad \mathcal{H} \\
& (u, v, x) \quad \mapsto \quad \pi_{X}\left(u, \zeta\left(v, w_{0}, x\right)\right)
\end{aligned}
$$

is with finite dimensional range;

(iii) $M\left(w_{0}\right)\left(u_{0}, v_{0}\right)=\left\{x_{0}\right\}$;

(iv) $\operatorname{deg}_{\mathcal{H}}\left(g_{X}\left(u_{0}, v_{0}, w_{0}, \cdot\right)\right.$, int $\left.X, 0\right) \neq 0$.

Then 
(a) $M\left(w_{0}\right)\left(u_{0}, v_{0}\right)=\left\{x_{0}\right\}$ and $N\left(w_{0}\right)\left(u_{0}, v_{0}\right)=\left\{z_{0}\right\}$;

(b) there exist a neighbourhood $U_{0} \subseteq U$ of $u_{0}$ and a neighbourhood $V_{0} \subseteq V$ of $v_{0}$ such that

$$
M\left(w_{0}\right)(u, v) \neq \emptyset \quad \text { and } \quad N\left(w_{0}\right)(u, v) \neq \emptyset, \quad \forall(u, v) \in U_{0} \times V_{0} ;
$$

(c) $M\left(w_{0}\right)$ and $N\left(w_{0}\right)$ are lsc at $\left(u_{0}, v_{0}\right)$.

Before giving the proof, it is worth noting that the assumption (iii) is equivalent to $N\left(w_{0}\right)\left(u_{0}, v_{0}\right)=\left\{z_{0}\right\}$, since by Theorem 3.4, $N\left(w_{0}\right)\left(u_{0}, v_{0}\right)=\left\{z_{0}\right\}$ if and only if $M\left(w_{0}\right)\left(u_{0}, v_{0}\right)=\left\{x_{0}\right\}$.

Proof. We will apply Theorem 2.7 with the mapping

$$
\begin{aligned}
f:(U \times V) \times \mathcal{H} & \rightarrow \mathcal{H} \\
(u, v, x) & \mapsto\left\{\begin{array}{l}
g_{X}\left(u, v, w_{0}, x\right) \text { if } x \in X \\
0 \text { otherwise. }
\end{array}\right.
\end{aligned}
$$

Observe first that, for all $(u, v, x) \in(U \times V) \times X$, we have

$$
\begin{aligned}
f(u, v, x) & =g_{X}\left(u, v, w_{0}, x\right) \\
& =x-\pi_{X}\left(u, w_{0}-\phi(v, x)+x\right) \\
& =x-\pi_{X}\left(u, \zeta\left(v, w_{0}, x\right)\right) .
\end{aligned}
$$

Setting $\Omega=\operatorname{int} X$, we have that, on $(U \times V) \times \bar{\Omega}, \pi_{X}\left(\cdot, \zeta\left(\cdot, w_{0}, \cdot\right)\right)$ is continuous by Hypotheses 1,2 and 3 , and with finite dimensional range by assumption (ii).

Now remark that, as $\mathcal{M}\left(w_{0}\right)=\mathcal{L}\left(w_{0}\right)$ by Theorem 3.3, we can rewrite the set-valued mapping

$$
\begin{aligned}
M\left(w_{0}\right): U \times V & \rightrightarrows X \\
(u, v) & \mapsto\left\{x \in X:\left(u, v, w_{0}, x\right) \in \mathcal{M}\left(w_{0}\right)\right\}
\end{aligned}
$$

as

$$
M\left(w_{0}\right)(u, v)=\left\{x \in X: g_{X}\left(u, v, w_{0}, x\right)=0\right\},
$$

i.e.,

$$
M\left(w_{0}\right)(u, v)=g_{X}\left(u, v, w_{0}, \cdot\right)^{-1}\{0\} \cap X .
$$

And by assumptions (iii) and (iv) we have $M\left(w_{0}\right)\left(u_{0}, v_{0}\right)=\left\{x_{0}\right\}$ and

$$
\operatorname{deg}_{\mathcal{H}}\left(f\left(u_{0}, v_{0}, \cdot\right), \Omega, 0\right)=\operatorname{deg}_{\mathcal{H}}\left(g_{X}\left(u_{0}, v_{0}, w_{0}, \cdot\right) ; \operatorname{int} X, 0\right) \neq 0 .
$$

It remains to verify that $0 \notin f\left(u_{0}, v_{0}\right.$, bd $\left.\Omega\right)$, i.e., $0 \notin g_{X}\left(u_{0}, v_{0}, w_{0}\right.$, bd $\left.\Omega\right)$.

If $0 \in g_{X}\left(u_{0}, v_{0}, w_{0}\right.$, bd $\left.\Omega\right)$, then there exist $x \in \operatorname{bd} \Omega$ such that $g_{X}\left(u_{0}, v_{0}, w_{0}, x\right)=0$, which means $x \in$ $M\left(w_{0}\right)\left(u_{0}, v_{0}\right)$, and so, by assumption (iii), $x=x_{0}$. Therefore we have that $x_{0} \notin \operatorname{int} X$ which contraddicts the fact that $X$ is a neighbourhood of $x_{0}$, hence $0 \notin g_{X}\left(u_{0}, v_{0}, w_{0}\right.$, bd $\left.\Omega\right)$.

We can now apply Theorem 2.7 to obtain that there exist a neighbourhood $U_{0} \subseteq U$ of $u_{0}$ and a neighbourhood $V_{0} \subseteq V$ of $v_{0}$ such that, $M\left(w_{0}\right)(u, v) \neq \emptyset$, for all $(u, v) \in U_{0} \times V_{0}$, and $M\left(w_{0}\right)$ is lsc at $\left(u_{0}, v_{0}\right)$.

This combined with Lemma 3.6 ensures that $N\left(w_{0}\right)$ is lsc at $\left(u_{0}, v_{0}\right)$ too.

Finally, we use Theorem 3.4 to prove (a) and (b). We start by showing that $N\left(w_{0}\right)\left(u_{0}, v_{0}\right)=\left\{z_{0}\right\}$.

As $x_{0} \in M\left(w_{0}\right)\left(u_{0}, v_{0}\right)$, we have $\left(u_{0}, v_{0}, w_{0}, x_{0}\right) \in \mathcal{M}\left(w_{0}\right)$, so by Theorem 3.4,

$$
\left(u_{0}, v_{0}, w_{0}, \zeta\left(v_{0}, w_{0}, x_{0}\right)\right) \in \mathcal{N}\left(w_{0}\right),
$$

i.e., $z_{0}:=\zeta\left(v_{0}, w_{0}, x_{0}\right) \in N\left(w_{0}\right)\left(u_{0}, v_{0}\right)$.

Now let $z \in N\left(w_{0}\right)\left(u_{0}, v_{0}\right)$. We have, once again by Theorem 3.4,

$$
z=\zeta\left(v_{0}, w_{0}, \pi_{X}\left(u_{0}, z\right)\right),
$$

with $\left(u_{0}, v_{0}, w_{0}, \pi_{X}\left(u_{0}, z\right)\right) \in \mathcal{M}\left(w_{0}\right)$. We deduce that $\pi_{X}\left(u_{0}, z\right) \in M\left(w_{0}\right)\left(u_{0}, v_{0}\right)$, which, by asumption (iii), implies that $\pi_{X}\left(u_{0}, z\right)=x_{0}$, and so, that

$$
z=\zeta\left(v_{0}, w_{0} x_{0}\right)=z_{0},
$$


hence $N\left(w_{0}\right)\left(u_{0}, v_{0}\right)=\left\{z_{0}\right\}$, which proves (a).

Let us show (b). We obtained that $M\left(w_{0}\right)(u, v) \neq \emptyset$, for all $(u, v) \in U_{0} \times V_{0}$, so for each $(u, v) \in U_{0} \times V_{0}$, there exist $x \in X$ such that $\left(u, v, w_{0}, x\right) \in \mathcal{M}\left(w_{0}\right)$. Thus, applying Theorem 3.4, we get

$$
\left(u, v, w_{0}, \zeta\left(v, w_{0}, x\right)\right) \in \mathcal{N}\left(w_{0}\right),
$$

that is, $\zeta\left(v, w_{0}, x\right) \in N\left(w_{0}\right)(u, v)$, hence $N\left(w_{0}\right)(u, v) \neq \emptyset$ which concludes the proof.

The next theorem establishes a similar result for the normal map.

Theorem 3.8. Let $u_{0} \in \mathcal{U}, v_{0} \in \mathcal{V}, w_{0}, x_{0} \in \mathcal{H}$ such that

$$
w_{0} \in N\left(C\left(u_{0}\right), x_{0}\right)+\phi\left(v_{0}, x_{0}\right) .
$$

Assume that there exist $U$, resp. $V, X$ and $Z$, neighbourhoods of $u_{0}$, resp. $v_{0}, x_{0}$ and $z_{0}:=\zeta\left(v_{0}, w_{0}, x_{0}\right)$ such that $U, V, W:=\left\{w_{0}\right\}, X$ and $Z$ satisfy Hypotheses 1, 2 and 3. Let $\mathcal{M}\left(w_{0}\right), \mathcal{N}\left(w_{0}\right)$ and $\mathcal{L}\left(w_{0}\right)$, and, $M\left(w_{0}\right)$ and $N\left(w_{0}\right)$ be as above.

Suppose also that:

(i) $Z$ is closed and bounded;

(ii)

$$
\begin{aligned}
U \times V \times Z & \rightarrow \mathcal{H} \\
(u, v, z) & \mapsto \pi_{X}(u, z)-\phi\left(v, \pi_{X}(u, z)\right)
\end{aligned}
$$

is with finite dimensional range;

(iii) $N\left(w_{0}\right)\left(u_{0}, v_{0}\right)=\left\{z_{0}\right\}$;

(iv) $\operatorname{deg}_{\mathcal{H}}\left(f_{X}\left(u_{0}, v_{0}, \cdot\right)\right.$, int $\left.Z, w_{0}\right) \neq 0$.

Then

(a) $M\left(w_{0}\right)\left(u_{0}, v_{0}\right)=\left\{x_{0}\right\}$ and $N\left(w_{0}\right)\left(u_{0}, v_{0}\right)=\left\{z_{0}\right\}$;

(b) there exist a neighbourhood $U_{0} \subseteq U$ of $u_{0}$ and a neighbourhood $V_{0} \subseteq V$ of $v_{0}$ such that

$$
M\left(w_{0}\right)(u, v) \neq \emptyset \quad \text { and } \quad N\left(w_{0}\right)(u, v) \neq \emptyset, \quad \forall(u, v) \in U_{0} \times V_{0} ;
$$

(c) $M\left(w_{0}\right)$ and $N\left(w_{0}\right)$ are lsc at $\left(u_{0}, v_{0}\right)$.

Once again we can change assumption (iii) into $M\left(w_{0}\right)\left(u_{0}, v_{0}\right)=\left\{x_{0}\right\}$, since, by Theorem 3.4, the two hypotheses are equivalent.

Proof. We apply Theorem 2.7 with the mapping

$$
\begin{aligned}
(U \times V) \times \mathcal{H} & \rightarrow \mathcal{H} \\
(u, v, z) & \mapsto\left\{\begin{array}{l}
f_{X}(u, v, z) \text { if } z \in Z \\
0 \text { otherwise }
\end{array}\right.
\end{aligned}
$$

to get the results on $N\left(w_{0}\right)$ and use Lemma 3.6 and Theorem 3.4 to obtain the results on $M\left(w_{0}\right)$ as we did in the proof of the Theorem 3.7.

\section{Prox-regularity and key hypotheses}

In section 3, all the results have been established under the assumption that $\Pi_{X}$ is single-valued on $U \times Z$ and its associated mapping $\pi_{X}$ continuous on $U \times Z$. Here we present a class of set-valued mappings that satisfy this assumption, the so-called prox-regular set -valued mapping with compatible parametrization.

We first give a short review of the theory of prox-regularity in order to give a definition of those so called prox-regular set-valued mappings. Then we will present the results showing single-valuedness and continuity of $\Pi_{X}$ under prox-regularity and continuity hypotheses on $C(\cdot)$. 


\subsection{Prox-regularity}

It is well known that in a Hilbert space $\mathcal{H}$ the metric projection mapping on a non-empty closed convex set $C$ is well-defined, that is, single-valued, and continuous on the entire space $\mathcal{H}$. Fortunately, this does not mean that if a non-empty closed set is not convex, then we cannot define the projection from no point of $\mathcal{H}$. Indeed, considering $S:=\mathbb{R}^{2} \backslash B(0 ; 1)$, the Euclidean plan without the unity open ball, we see that the projection on $S$ of the origin point is multi-valued, but single-valued for every other point in $B(0,1) \backslash\{0\}$. Therefore, given $0<r<1$, we can define the $r$-enlargement of $S$,

$$
E_{r}(S):=\left\{x \in \mathbb{R}^{2}: d_{S}(x) \leq r\right\}
$$

on which $P_{S}(\cdot)$ is single-valued.

This notion has first been considered by Federer in 1959 [7] in which he defines $r$-positively reached sets $S$ in $\mathbb{R}^{N}$ as non-empty closed sets of $\mathbb{R}^{N}$ satisfying uniqueness of the projection mapping on some $r$-enlargement of $S, r$ being a positive real. Similar concepts appeared in the literature under other names and forms (see [22], $[2],[20],[3],[14]$ and [4]) and are nowaday known to coincide under the name of (uniformly) $r$-prox-regular sets.

Now if $S \subseteq \mathbb{R}^{2}$ is the complementary of a triangle in $\mathbb{R}^{2}$, we see that $S$ is not uniformly prox-regular because at any point of one of the bisectors that does not belong to $S$, we will always have two projections on $S$. Hence, there is no $r>0$ such that the projection mapping is well-defined on the $r$-enlargement of $S$. However, for every point of the boundary of $S$ except the vertices, we can find a neighbourhood of the point on which the projection is well-defined and continuous on a certain way. This is what Poliquin, Rockafellar and Thibault [14] called local prox-regularity. Thus, a non-empty closed set $S$ of a Hilbert space $\mathcal{H}$ is (locally) prox-regular at a point $\bar{x} \in S$ if $P_{S}(\cdot)$ is single-valued and strongly-weakly continuous on a neighbourhood of $\bar{x}$.

This property is equivalent to the following analytic definition.

Definition 4.1. A nonempty closed set $S \subseteq \mathcal{H}$ is prox-regular at $\bar{x} \in S$ if there exists $\varepsilon, \rho>0$ such that for all $x, x^{\prime} \in S \cap B(\bar{x}, \varepsilon)$, and all $v \in N^{L}(S, x) \cap B(0, \varepsilon)$, we have

$$
\left\langle v, x^{\prime}-x\right\rangle \leq \frac{\rho}{2}\left|x^{\prime}-x\right|^{2} .
$$

Remark that in Definition 4.1, the notation $N^{L}(S, x)$ refers to the Mordukhovich normal cone, or limiting normal, of the set $S$ at the point $x$ (see [12] for definition and properties). However it is known (see [5]) that when Definition 4.1 holds for $N^{P}(S, x), N^{F}(S, x)$, or $N^{L}(S, x)$ of $N^{C l}(S, x)$, refering respectively to the proximal normal cone, the Frechet normal cone, the Mordukhovich normal cone and the Clarke normal cone (see [12] for definitions and properties), then one has

$$
N^{P}(S, x)=N^{F}(S, x)=N^{L}(S, x)=N^{C l}(S, x) .
$$

Under this consideration, from now on the notation $N(S, x)$ will refer to one of those four normal cones.

Definition 4.1 is related to the definition of Poliquin and Rockafellar [13] for prox-regular functions to $\Psi_{S}$ the indicator function of $S$.

We refer the reader to [14] and [5] for more results and history on local and uniform prox-regularity.

Then Robinson [18] extended the concept of local prox-regularity to parameterized sets $\{C(u)\}_{u \in \mathcal{U}}$ in the finite dimensional setting by applying the definition of local prox-regular parameterized functions given by Levy, Poliquin and Rockafellar [11] to the family of functions $\left\{\Psi_{C(u)}\right\}_{u \in \mathcal{U}}$. This gives the following definition in the general Hilbert case.

Definition 4.2. Given a nonempty closed valued set-valued mapping $C(\cdot)$ from a topological space $\mathcal{U}$ to a Hilbert space $\mathcal{H}$, a point $\bar{u} \in \mathcal{U}$ and a point $\bar{x} \in C(\bar{u})$, we say that $C(\cdot)$ is prox-regular in $x$ at $\bar{x}$ with compatible parametrization by $u$ at $\bar{u}$ if there exist a neighbourhood $U$ of $\bar{u}$ and $\varepsilon, \rho>0$ such that, for all $u \in U$, all $x, x^{\prime} \in C(u) \cap B(\bar{x}, \varepsilon)$ and all $v \in N(C(u), x) \cap B(0, \varepsilon)$, we have

$$
\left\langle v, x^{\prime}-x\right\rangle \leq \frac{\rho}{2}\left|x^{\prime}-x\right|^{2} .
$$

We are now able to see how prox-regularity satisfies the assumptions on $\Pi_{X}$. 


\subsection{Single-valuedness and continuity of $\Pi_{X}$}

In a previous work [21], we showed single-valuedness and continuity of a localization of $\Pi_{X}$ under proxregularity and continuity assumptions on $C(\cdot)$. We briefly recall those theorems in order to apply them to our stability problems. We refer the reader to [21] for the proofs and more discussions on the results.

The first result shows that under prox-regularity the set-valued mapping $\Pi_{X}$ is single-valued on a localization and Lipschitz continuous with respect to the second variable with a certain control on the Lipschitz constant. But first we need to recall what lower semicontinuity of a set-valued mapping is.

Definition 4.3. Recall that the set-valued mapping $C: \mathcal{U} \rightrightarrows \mathcal{H}$ is inner semicontinuous at $\bar{u} \in \mathcal{U}$ for $\bar{x} \in C(\bar{u})$ whenever for each neighbourhood $X$ of $\bar{x}$ there is a neighbourhood $U$ of $\bar{u}$ such that $X \cap C(u) \neq \emptyset$ for all $u \in U$.

Theorem 4.4 ([21]). Let $C: \mathcal{U} \rightrightarrows \mathcal{H}$ be a set-valued mapping with nonempty closed values, $\bar{u}$ be a point in $\mathcal{U}$, and $\bar{x}$ be a point in $H$ with $\bar{x} \in C(\bar{u})$. Assume that $C(\cdot)$ is inner semicontinuous at $\bar{u}$ for $\bar{x}$ and that $C(\cdot)$ is also prox-regular in $x$ at the point $\bar{x}$ with compatible parametrization by $u$ at $\bar{u}$.

Then for any real $\beta>1$ there exist an open neighbourhood $U$ of $\bar{u}$ and two closed neighbourhoods $X$ and $Z$ of $\bar{x}$ such that

(a) for all $u \in U$, the set-valued mapping

$$
\begin{aligned}
\Pi_{X}(u, \cdot): \quad H & \rightrightarrows H \\
z & \mapsto\left(N^{L}(C(u), \cdot)+I\right)^{-1}(z) \cap X
\end{aligned}
$$

is single valued and Lipschitz continuous on $Z$ with $\beta$ as a Lipschitz constant;

(b) for all $u \in U$, the mapping $P_{C(u)}(\cdot)$ is well-defined on $X$, that is, $\operatorname{Proj}_{C(u)}(\cdot)$ is nonempty and single valued on $X$;

(c) for all $u \in U$ and $z \in Z$,

$$
\Pi_{X}(u, z)=P_{C(u)}(z)
$$

The second result establishes a quantitative bound of $\Pi_{X}$ with respect to the first variable involving the $\sigma$-Hausdorff semidistance.

When dealing with distances between closed sets, a key concept is the excess between two closed sets $S, S^{\prime} \subseteq \mathcal{H}$, defined by

$$
e\left(S, S^{\prime}\right)=\sup _{x \in S} d_{S^{\prime}}(x)
$$

Since usually $e\left(S, S^{\prime}\right) \neq e\left(S^{\prime}, S\right)$, we create distances by taking the maximum between the two quantities. For instance, the Pompeiu-Hausdorff distance between the closed sets $S$ and $S^{\prime}$ is given by

$$
\text { haus }\left(S, S^{\prime}\right)=\max \left\{e\left(S, S^{\prime}\right), e\left(S^{\prime}, S\right)\right\}
$$

However, it can be easily seen that, when dealing with unbounded sets, the Hausdorff distance may be infinite. To avoid the problem, the idea is to truncate the sets. Thus, given $\sigma>0$, we define the $\sigma$-Hausdorff semidistance between closed sets $S$ and $S^{\prime}$ by

$$
\operatorname{haus}_{\sigma}\left(S, S^{\prime}\right)=\max \left\{e\left(S \cap \sigma \mathbb{B}, S^{\prime}\right), e\left(S^{\prime} \cap \sigma \mathbb{B}, S\right)\right\} \text {, }
$$

and the $(\sigma, \sigma)$-Hausdorff semidistance by

$$
\operatorname{haus}_{\sigma, \sigma}\left(S, S^{\prime}\right)=\max \left\{e\left(S \cap \sigma \mathbb{B}, S^{\prime} \cap \sigma \mathbb{B}\right), e\left(S^{\prime} \cap \sigma \mathbb{B}, S \cap \sigma \mathbb{B}\right)\right\} .
$$

The fact that

$$
\operatorname{haus}_{\sigma}\left(S, S^{\prime}\right) \leq \operatorname{haus}\left(S, S^{\prime}\right) \quad \text { and } \operatorname{haus}_{\sigma}\left(S, S^{\prime}\right) \leq \operatorname{haus}_{\sigma, \sigma}\left(S, S^{\prime}\right)
$$

motivated the choice of haus $\sigma\left(S, S^{\prime}\right)$ in the next result. 
Theorem 4.5 ([21]). Let $C: \mathcal{U} \rightrightarrows \mathcal{H}$ be a set-valued mapping with nonempty closed values, $\bar{u}$ be a point in $\mathcal{U}$, and $\bar{x}$ be a point in $\mathcal{H}$ with $\bar{x} \in C(\bar{u})$. Assume that $C(\cdot)$ is inner semicontinuous at $\bar{u}$ for $\bar{x}$ and that $C(\cdot)$ is also prox-regular in $x$ at the point $\bar{x}$ with compatible parametrization by $u$ at $\bar{u}$.

Let $\beta>1$ and let $U, X, Z$ and $\Pi_{X}$ be as in Theorem 4.4. Reduce the neighbourhood $X$ of $\bar{x}$ to be bounded and choose $\sigma>0$ such that $X \subseteq \sigma \mathbb{B}$.

Then, for all $z \in Z$ and all $u, u^{\prime} \in U$, we have

$$
\left|\Pi_{X}(u, z)-\Pi_{X}\left(u^{\prime}, z\right)\right| \leq \frac{h \beta}{2}+\left[2 h \beta\left|z-\Pi_{X}(u, z)\right|+h^{2}\left(\frac{\beta^{2}}{4}+\beta-1\right)\right]^{\frac{1}{2}}
$$

where $h=\operatorname{haus}_{\sigma}\left(C(u), C\left(u^{\prime}\right)\right)$.

Now we would like that, for $u$ tending to $\bar{u}, \operatorname{haus}_{\sigma}(C(u), C(\bar{u}))$ tends to 0 in order to obtain continuity of $\Pi_{X}$ with respect to the first variable. But since the choice of $\sigma$ in Theorem 4.5 is determined by the size of $X$ which is not known a priori, we want " $u$ close to $\bar{u} \operatorname{implies~haus~}_{\sigma}(C(u), C(\bar{u}))$ close to 0 " for all $\sigma>0$. This concept is known under the name of Attouch-Wets continuity (see [1] and [15]).

Definition 4.6. Let $C(\cdot)$ be a set-valued mapping from $\mathcal{U}$ into $\mathcal{H}$ with nonempty closed values and $\bar{u}$ be a point in $\mathcal{U}$. One says that $C(\cdot)$ is Attouch-Wets continuous at $\bar{u}$ or continuous for the $\sigma$-Hausdorff semidistances if for any $\sigma \geq 0$ and any $\varepsilon>0$ there exists a neighbourhood $U$ of $\bar{u}$ such that for all $u \in U$, haus $\sigma(C(u), C(\bar{u}))<\varepsilon$.

If now $U$ is an open subset of $T$, we will say that $C(\cdot)$ is Attouch-Wets continuous on $U$ or continuous on $U$ for the $\sigma$-Hausdorff semidistances if the corresponding continuity property for $C(\cdot)$ is fulfilled at each point $u \in U$.

We can then establish the continuity of $\Pi_{X}$ with respect to the first variable which, by uniform continuity of $\Pi_{X}$ with respect to the second variable, implies that $\Pi_{X}$ is continuous with respect to the pair $(u, z)$.

Theorem 4.7 ([21]). Let $C: \mathcal{U} \rightrightarrows \mathcal{H}$ be a set-valued mapping with nonempty closed values, $\bar{u}$ be a point in $\mathcal{U}$, and $\bar{x}$ be a point in $\mathcal{H}$ with $\bar{x} \in C(\bar{u})$. Assume that $C(\cdot)$ is prox-regular in $x$ at $\bar{x}$ with compatible parametrization by $u$ at $\bar{u}$. Assume also that $C(\cdot)$ is Attouch-Wets continuous on a neighbourhood $O$ of $\bar{u}$, i.e., continuous with respect to the family of $\sigma$-Hausdorff semidistances.

Let $\beta>1$ and let $U, X, Z$ be as given by Theorem 4.5. Then the single valued mapping

$$
\begin{aligned}
\Pi_{X}: U \times Z & \rightarrow H \\
(u, z) & \mapsto\left(N^{L}(C(u), \cdot)+I\right)^{-1}(z) \cap X
\end{aligned}
$$

is continuous on $(U \cap O) \times Z$. Further

$$
\Pi_{X}(u, z)=P_{C(u)}(z) \quad \text { for all } u \in U \cap O \text { and } z \in Z \text {. }
$$

Therefore we have given a sufficient condition for $\Pi_{X}$ to be single-valued and continuous on a localization.

\subsection{Relaxed hypotheses}

Practically, without any assumption on the way that $C(\cdot)$ evolves under a variation of $u$, it can be very difficult to find sets $X$ and $Z$ satisfying Hypotheses 1 and 2 .

However, we can slightly relax those assumptions and still have $\mathcal{M}=\mathcal{L}$ and $\mathcal{M}$ homeomorph to $\mathcal{N}$.

Hypothesis $\mathbf{1}^{*}$. We suppose that there exist sets $U \subseteq \mathcal{U}$ and $X, Z \subseteq \mathcal{H}$ such that the set-valued mapping

$$
\begin{aligned}
& \Pi_{X}: \quad U \times Z \quad \rightrightarrows X \\
& (u, z) \quad \mapsto \quad(N(C(u), \cdot)+I)^{-1} \cap X
\end{aligned}
$$

admits a continuous selection $\pi_{X}$ on $U \times Z$.

Let us recall now that Hypothesis 2 was due to the fact that

$$
w \in N(C(u), x)+\phi(v, x) \Leftrightarrow x \in(N(C(u), \cdot)+I)^{-1}(x+w-\phi(v, x)) .
$$


However, as Kien and Yao observed in [10], this equivalence is a particular case of the following one:

$$
w \in N(C(u), x)+\phi(v, x) \Leftrightarrow x \in(N(C(u), \cdot)+I)^{-1}(x+(\delta(w-\phi(v, x)))), \quad \forall \delta>0,
$$

which holds true according to the cone property of $N(C(u), \cdot)$. We can then transform Hypothesis 2 into the following hypothesis.

Hypothesis $2^{*}$. Under the notations of Hypothesis $1^{*}$, we suppose that there exist $\delta>0, V \subseteq \mathcal{V}$ and $W \in \mathcal{H}$ such that

$$
\zeta^{\delta}(v, w, x):=x+\delta(w-\phi(v, x)) \in Z, \quad \forall(v, w, x) \in V \times W \times X
$$

So far, we can define the generalized natural and normal mappings and the sets of solutions as follows.

Definition 4.8. Let $\delta>0, U \subseteq \mathcal{U}, V \subseteq \mathcal{V}$ and $W, X, Z \subseteq \mathcal{H}^{3}$ satisfying Hypotheses $1^{*}$ and $2^{*}$ and define $\Pi_{X}$ and $\pi_{X}$ as in Hypothesis $1^{*}$.

We define the generalized local normal map by

$$
\begin{aligned}
f_{X}^{*}: U \times V \times Z & \rightarrow \mathcal{H}, \\
(u, v, z) & \mapsto \frac{1}{\delta}\left(z-\pi_{X}(u, z)\right)+\phi\left(v, \pi_{X}(u, z)\right)
\end{aligned}
$$

and the generalized local natural map by

$$
\begin{aligned}
g_{X}^{*}: U \times V \times W \times X & \rightarrow \mathcal{H} \\
(u, v, w, z) & \mapsto x-\pi_{X}(u, x+\delta(w-\phi(v, x))) .
\end{aligned}
$$

Definition 4.9. Let $\delta>0, U \subseteq \mathcal{U}, V \subseteq \mathcal{V}$ and $W, X, Z \subseteq \mathcal{H}^{3}$ satisfying Hypotheses $1^{*}, 2^{*}$ and define $\Pi_{X}$ and $\pi_{X}$ as in Hypothesis $1^{*}$.

We define

$$
\begin{gathered}
\mathcal{M}^{*}:=\{(u, v, w, x) \in U \times V \times W \times X: w \in N(C(u), x)+\phi(v, x), \\
\mathcal{N}^{*}:=\left\{(u, v, w, z) \in U \times V \times W \times Z: w=f_{X}^{*}(u, v, x)\right\},
\end{gathered}
$$

and

$$
\mathcal{L}^{*}:=\left\{(u, v, w, x) \in U \times V \times W \times X: 0=g_{X}^{*}(u, v, w, x)\right\} .
$$

To keep the same relations between those sets, we have to introduce another hypothesis. Indeed, we recall that in the last section we had $\mathcal{M} \subseteq \mathcal{L}$ because

$$
\begin{aligned}
(u, v, w, x) \in \mathcal{M} & \Rightarrow x \in \Pi_{X}(u, \zeta(v, w, x)) \\
& \Rightarrow x=\pi_{X}(u, \zeta(v, w, x))
\end{aligned}
$$

since $\Pi_{X}$ was supposed single-valued.

But since now $\Pi_{X}$ is not necessarily single-valued, there are no reason to have $x=\pi_{X}(u, \zeta(v, w, x))$, so we need to assume it.

Hypothesis $3^{*}$. Let $\delta>0, U \subseteq \mathcal{U}, V \subseteq \mathcal{V}$ and $W, X, Z \subseteq \mathcal{H}^{3}$ satisfying Hypotheses $1^{*}, 2^{*}$ and define $\Pi_{X}$ and $\pi_{X}$ as in Hypothesis $1^{*}$.

We suppose that for $(u, v, w, x) \in \mathcal{M}$, one has

$$
x=\pi_{X}\left(u, \zeta^{\delta}(v, w, x)\right) .
$$

And finally, we have the following hypothesis to have $f_{X}^{*}$ and $g_{X}^{*}$ continuous.

Hypothesis $4^{*}$. Let $\delta>0, U \subseteq \mathcal{U}, V \subseteq \mathcal{V}$ and $W, X, Z \subseteq \mathcal{H}^{3}$ satisfying Hypotheses $1^{*}, 2^{*}$.

We suppose that $\phi$ is continuous on $V \times X$.

The next two theorems show that the links between the sets $\mathcal{M}^{*}, \mathcal{N}^{*}$ and $\mathcal{L}^{*}$ are the same than the one between $\mathcal{M}, \mathcal{N}$ and $\mathcal{L}$ in the previous section. 
Theorem 4.10. Let $\delta>0, U \subseteq \mathcal{U}, V \subseteq \mathcal{V}$ and $W, X, Z \subseteq \mathcal{H}$ satisfying Hypotheses $1^{*}$, 2*, 3* and $4^{*}$, and define $\Pi_{X}$ and $\pi_{X}$ as in Hypothesis $1^{*}$, and, $\mathcal{M}^{*}$ and $\mathcal{L}^{*}$ as in Definition 4.9.

Then one has

$$
\mathcal{M}^{*}=\mathcal{L}^{*}
$$

Proof. By Hypothesis $3^{*}$, it is clear that $\mathcal{M}^{*} \subseteq \mathcal{L}^{*}$.

Now let us take $(u, v, w, x) \in \mathcal{L}^{*}$, then $(u, v, w, x) \in U \times V \times W \times X$, with

$$
x=\pi_{X}\left(u, \zeta^{\delta}(v, w, x)\right) .
$$

So by definitions of $\pi_{X}$ and $\zeta^{\delta}$, one has

$$
x \in(N(C(u), \cdot)+I)^{-1}(x+\delta(w+\phi(v, x))),
$$

which implies that

$$
x+\delta(w+\phi(v, x)) \in N(C(u), x)+x,
$$

i.e.,

$$
\delta(w+\phi(v, x)) \in N(C(u), x) .
$$

Now, $N(C(u), x)$ is a cone and $\delta>0$, we deduce

$$
w+\phi(v, x) \in N(C(u), x),
$$

or equivalently,

$$
w \in N(C(u), x)+\phi(v, x),
$$

which gives $(u, v, w, x) \in \mathcal{L}^{*}$ and concludes the proof.

Likewise, the sets $\mathcal{M}^{*}$ and $\mathcal{N}^{*}$ are still homeomorph.

Theorem 4.11. Let $\delta>0, U \subseteq \mathcal{U}, V \subseteq \mathcal{V}$ and $X, Z \subseteq \mathcal{H}$ be as in Hypotheses $1^{*}, 2^{*}, 3^{*}$ and $4^{*}$, and define $\pi_{X}$ as in Hypothesis $1^{*}, f_{X}^{*}$ as in Definition $4.8, \zeta^{\delta}$ as in $2^{*}$, and $\mathcal{M}^{*}, \mathcal{N}^{*}$ and $\mathcal{L}^{*}$ as in Definition 4.9 .

Then the map

$$
\begin{aligned}
& \mu^{*}: \mathcal{M}^{*} \quad \rightarrow \mathcal{N}^{*} \\
& (u, v, w, x) \mapsto\left(u, v, w, \zeta^{\delta}(v, w, x)\right)
\end{aligned}
$$

is an homeomorphism from $\mathcal{M}^{*}$ to $\mathcal{N}^{*}$, and its inverse map is given by

$$
\begin{aligned}
& \nu^{*}: \mathcal{N}^{*} \quad \rightarrow \mathcal{M}^{*} \\
& (u, v, w, z) \mapsto\left(u, v, w, \pi_{X}(u, z)\right) .
\end{aligned}
$$

Proof. Similar to the proof of Theorem 3.4.

We now see that those hypotheses are fully fulfilled by the concept of uniform prox-regularity.

\subsection{Uniform prox-regularity and Hypotheses $1^{*}, 2^{*}, 3^{*}$ and $4^{*}$}

Let us recall that a non-empty closed $S$ is (uniform) prox-regular if there exists a real $r>0$ such that the projection mapping $P_{S}(\cdot)$ is single-valued and strongly-weakly continuous on the $r$-enlargement of $S$,

$$
E_{r}(S):=\left\{x \in \mathbb{R}^{2}: d_{S}(x) \leq r\right\} .
$$

This definition can be stated in the following analytic equivalent form.

Definition 4.12. Given a non-empty closed set $S \subseteq \mathcal{H}$, we say that $S$ is (uniformly) prox-regular if there exists $\rho>0$ such that, for all $x, x^{\prime} \in S$, and all $v \in N(S, x) \cap \mathbb{B}$, one has

$$
\left\langle v, x^{\prime}-x\right\rangle \leq \frac{\rho}{2}\left|x^{\prime}-x\right|^{2}
$$

where $\mathbb{B}$ denotes the closed unit ball centered at the origin. 
Like for the concept of local prox-regularity seen in the begining of this section, we can extend the concept of uniform prox-regularity to set-valued mappings by the following definition.

Definition 4.13. Given a set-valued mapping $C: \mathcal{U} \rightrightarrows \mathcal{H}$ with non-empty closed values and a point $\bar{u} \in$ $\mathcal{U}$, we say that $C(\cdot)$ is (uniformly) prox-regular with compatible parametrization by $u$ at $\bar{u}$ if there exists a neighbourhood $U$ of $\bar{u}$ and a real $\rho>0$ such that for all $u \in U$, all $x, x^{\prime} \in C(u)$ and all $v \in N(C(u), x) \cap \mathbb{B}$, one has

$$
\left\langle v, x^{\prime}-x\right\rangle \leq \frac{\rho}{2}\left|x^{\prime}-x\right|^{2} .
$$

The following proposition links the concepts of local and uniform prox-regularity.

Proposition 4.14. If $C(\cdot)$ is (uniformly) prox-regular with compatible parametrization by $u$ at $\bar{u}$ for the parameters $U$ and $\rho$, then $C(\cdot)$ is (locally) prox-regular for $x$ at $\bar{x}$ with compatible parametrization by $u$ at $\bar{u}$ for all $\bar{x} \in C(\bar{u})$ and for the parameters $U, \rho$ and $\varepsilon \leq 1$.

We can then extend Theorem 4.7 to the setting of uniform prox-regularity. But first we need to express Theorem 4.7 in the following quantitative way. Indeed, the proof of Theorem 4.7 shows the following result.

Theorem 4.15. Let $C: \mathcal{U} \rightrightarrows \mathcal{H}$ be a set valued-mapping with non-empty closed values, $\bar{u} \in \mathcal{U}$ and $\bar{x} \in C(\bar{u})$. Suppose

(i) $C(\cdot)$ is (locally) prox-regular for $x$ at $\bar{x}$ with compatible parametrization by $u$ at $\bar{u}$ for the parameters $U$, $\rho>0$ and $\varepsilon>0$

(ii) $C(\cdot)$ is Attouch-Wets continuous on a neighbourhood $O$ of $\bar{u}$.

Then, for $\beta>1$, choosing $\gamma_{1}, \gamma_{2}, \eta>0$ such that

$$
\left\{\begin{array}{l}
\gamma_{1}<\min \left\{\frac{\varepsilon}{2}, \frac{1-\beta^{-1}}{2 \rho} \varepsilon\right\} \\
\eta<\min \left\{\gamma_{1}, \varepsilon-2 \gamma_{1}\right\} \\
\eta+2 \gamma_{2}<\gamma_{1}
\end{array}\right.
$$

and setting $X_{0}:=\bar{B}\left(\bar{x}, \gamma_{1}\right), Z_{0}:=\bar{B}\left(\bar{x}, \gamma_{2}\right)$ and $U_{0}:=\left\{u \in U \cap O: d_{C(u)}(\bar{x})<\eta\right\}$, we have:

(a) the set-valued mappings

$$
\Pi_{X_{0}}:(u, z) \mapsto(N(C(u), \cdot)+I)^{-1}(z) \cap X_{0} \quad \text { and } \quad P_{C}:(u, z) \mapsto P_{C(u)}(z)
$$

are single-valued and continuous on $U_{0} \times Z_{0}$;

(b) $\Pi_{X_{0}}(u, z)=\left\{P_{C(u)}(z)\right\}$, for all $(u, z) \in U_{0} \times Z_{0}$.

Thanks to Proposition 4.14, we can extend Theorem 4.15.

Theorem 4.16. Let $C: \mathcal{U} \rightrightarrows \mathcal{H}$ be a set valued-mapping with non-empty closed values and $\bar{u} \in \mathcal{U}$.

Suppose

(i) $C(\cdot)$ is (uniformly) prox-regular with compatible parametrization by $u$ at $\bar{u}$ for the parameters $U$ and $\rho>0$;

(ii) $C(\cdot)$ is Attouch-Wets continuous on a neighbourhood $O$ of $\bar{u}$;

(iii) $C(\cdot)$ is Hausdorff continuous at $\bar{u}$.

Then, for $\beta>1$ and $\varepsilon \leq 1$, choosing $\gamma_{1}, \gamma_{2}, \eta>0$ as in Theorem 4.15 and setting

$$
\begin{gathered}
U_{0}:=\{u \in U \cap O: \text { haus }(C(u), C(\bar{u}))<\eta\}, \\
X_{0}:=\left\{x \in \mathcal{H}: d_{C(\bar{u})}(x) \leq \gamma_{1}\right\}
\end{gathered}
$$

and

$$
Z_{0}:=\left\{x \in \mathcal{H}: d_{C(\bar{u})}(x) \leq \gamma_{2}\right\}
$$

one has $P_{C}:(u, z) \mapsto P_{C(u)}(z)$ is single-valued and continuous on $U_{0} \times Z_{0}$ and for all $(u, z) \in U_{0} \times Z_{0}$, $P_{C(u)}(z) \in \Pi_{X_{0}}(u, z)$, in other words, $P_{C}$ is a continuous selection of $\Pi_{X_{0}}$ on $U_{0} \times Z_{0}$. 
Proof. Let $\beta>1$ and $\varepsilon \leq 1$ and choose $\gamma_{1}, \gamma_{2}, \eta>0$ as in Theorem 4.15. Choose also $\gamma_{0}>0$ such that $\gamma_{0}>\gamma_{2}$ and $2 \gamma_{0}+\eta<\gamma_{1}$.

As $C(\cdot)$ is prox-regular at $\bar{u}$ for the parameters $U$ and $\rho$, we have by Proposition 4.14, that for all $x \in C(\bar{u})$, $C(\cdot)$ is (locally) prox-regular for $x$ at $\bar{x}$ with compatible parametrization by $u$ at $\bar{u}$ for the parameters $U, \rho$ and $\varepsilon$.

So, setting for each $x \in C(\bar{u}), X(x):=\bar{B}\left(x, \gamma_{1}\right), Z(x):=\bar{B}\left(x, \gamma_{0}\right)$ and $U(x):=\left\{u \in U \cap O: d_{C(u)}(x)<\eta\right\}$, we have by Theorem 4.15 that $P_{C}$ is single-valued and continuous on $U(x) \times Z(x)$ with $P_{C(u)}(x) \in \Pi_{X(x)}(u, z)=$ $(N(C(u), \cdot)+I)^{-1}(z) \cap X(x)$, for all $\left.u, z\right) \in U(x) \times Z(x)$.

Set

$$
\begin{aligned}
U_{0}:= & \{u \in U \cap O: \text { haus }(C(u), C(\bar{u}))<\eta\}, \\
& X_{0}:=\left\{x \in \mathcal{H}: d_{C(\bar{u})}(x) \leq \gamma_{1}\right\}
\end{aligned}
$$

and

$$
Z_{0}:=\left\{x \in \mathcal{H}: d_{C(\bar{u})}(x) \leq \gamma_{2}\right\}
$$

Let us show that $P_{C}$ is single-valued on $U_{0} \times Z_{0}$ with $P_{C(u)}(z) \in \Pi_{X_{0}}(u, z)$ for all $(u, z) \in U_{0} \times Z_{0}$.

Let $(u, z) \in U_{0} \times Z_{0}$. As $z \in Z_{0}$ and $\gamma_{0}>\gamma_{1}$, there exists $\bar{x} \in C(\bar{u})$ such that $z \in \bar{B}\left(\bar{x}, \gamma_{0}\right)=Z(\bar{x})$. And as $\bar{x} \in C(\bar{u})$, we have por all $u \in U_{0}$ that

$$
d_{C(u)}(\bar{x}) \leq \text { haus }(C(u), C(\bar{u}))<\eta,
$$

(remark here that $U_{0}$ is non-empty by the Hausdorff continuity of $C(\cdot)$ at $\bar{u}$ ).

So, by what precedes, one has that $P_{C(u)}(z)$ is well-defined and continuous at $(u, z)$.

Moreover, we have that

$$
P_{C(u)}(z) \in \Pi_{X(\bar{x})}(u, z)=(N(C(u), \cdot)+I)^{-1}(z) \cap X(\bar{x}) .
$$

But since

$$
X(\bar{x})=\bar{B}\left(\bar{x}, \gamma_{1}\right) \subseteq\left\{x \in \mathcal{H}: d_{C(\bar{u})}(x) \leq \gamma_{1}\right\}=X_{0},
$$

as $\bar{x} \in C(\bar{u})$, we deduce that $P_{C(u)}(z) \in \Pi_{X_{0}}(u, z)$ which proves the result.

We will also need the following proposition.

Proposition 4.17. If $C(\cdot)$ is (uniformly) prox-regular with compatible parametrization by $u$ at $\bar{u}$ for the parameters $U$ and $\rho$, then for all $u \in U$, all $x \in C(u)$ and all $v \in N(C(u), x) \cap \mathbb{B}$,

$$
x \in P_{C(u)}\left(x+\frac{2}{\rho} v\right) .
$$

Proof. Let $u \in U, x \in C(u)$ and $v \in N(C(u), x) \cap \mathbb{B}$. Then, for all $x^{\prime} \in C(u)$, one has

$$
\left\langle v, x^{\prime}-x\right\rangle \leq \frac{\rho}{2}\left|x^{\prime}-x\right|^{2},
$$

so

$$
0 \leq\left\langle\frac{2}{\rho} v, x-x^{\prime}\right\rangle+\left|x-x^{\prime}\right|^{2} .
$$

Hence, adding $\left|\frac{2}{\rho} v\right|^{2}$ to both sides of the inequalities, we obtain for all $x^{\prime} \in C(u)$,

$$
\begin{aligned}
\left|\left(x+\frac{2}{\rho}\right)-x\right|^{2} & =\left|\frac{2}{\rho} v\right|^{2} \\
& \leq\left|\frac{2}{\rho} v\right|^{2}+\left\langle\frac{2}{\rho} v, x-x^{\prime}\right\rangle+\left|x-x^{\prime}\right|^{2} \\
& =\left|\left(x+\frac{2}{\rho}\right)-x^{\prime}\right|^{2}
\end{aligned}
$$

which implies that

$$
\left|\left(x+\frac{2}{\rho}\right)-x\right| \leq\left|\left(x+\frac{2}{\rho}\right)-x^{\prime}\right|, \quad \forall x^{\prime} \in C(u),
$$

and gives $x \in P_{C(u)}\left(x+\frac{2}{\rho} v\right)$ as wanted. 
We can now show that under uniform prox-regularity and continuity of $C(\cdot)$, Hypotheses $1^{*}, 2^{*}, 3^{*}$ and $4^{*}$ are fulfilled.

Theorem 4.18. Let $w_{0}, x_{0} \in \mathcal{H}, u_{0} \in \mathcal{U}$ and $v_{0} \in \mathcal{V}$ such that

$$
w_{0} \in N\left(C\left(u_{0}\right), x_{0}\right)+\phi\left(v_{0}, x_{0}\right)
$$

\section{Suppose}

(i) $C(\cdot)$ is (uniformly) prox-regular at $u_{0}$ for the parameters $\rho>0$ and $U$;

(ii) $C(\cdot)$ is Attouch-Wets continuous on a neighbourhood $O$ of $u_{0}$;

(iii) $C(\cdot)$ is Hausdorff continuous at $u_{0}$;

(iv) $\phi$ is continuous and bounded on $V \times X$ where $V$ is a neighbourhood of $v_{0}$ and $X=\left\{x \in \mathcal{H}: d_{C\left(u_{0}\right)}(x) \leq \gamma\right\}$ for some $\gamma>0$.

Then, for any $\alpha>0$, there exist $\delta>0$, neighbourhoods $U_{0}$, resp. $V_{0}, W_{0}$ and $X_{0}$, of $u_{0}$, resp. $v_{0}$, $w_{0}$ and $x_{0}$, and a set $Z_{0}$ such that $\delta, U_{0}, V_{0}, W_{0}, X_{0}$ and $Z_{0}$ satisfy Hypotheses $1^{*}, 2^{*}$, $3^{*}$ and $4^{*}$ with $X_{0}$ and $Z_{0}$ closed, $Z_{0}$ neighbourhood of $\zeta^{\delta}\left(v_{0}, w_{0}, x_{0}\right)$ and $W_{0}:=\bar{B}\left(w_{0}, \alpha\right)$.

Proof. Let $0<\varepsilon \leq 1$ and $\beta>1$. Let us take $\gamma_{1}, \gamma_{2}, \eta>0$ as in Theorem 4.15. Set

$$
U_{0}:=\left\{u \in U \cap O: \text { haus }\left(C(u), C\left(u_{0}\right)\right)<\min \left\{\frac{\gamma_{2}}{2}, \eta, \gamma\right\}\right\},
$$

which is a neighbourhood of $u_{0}$ as $C(\cdot)$ is Hausdorff continuous at $u_{0}$. Set also

$$
\begin{gathered}
Z_{0}:=\left\{x \in \mathcal{H}: d_{C\left(u_{0}\right)}(x) \leq \gamma_{2}\right\}, \\
X_{0}:=\left\{x \in \mathcal{H}: d_{C\left(u_{0}\right)}(x) \leq \min \left\{\frac{\gamma_{2}}{2}, \gamma\right\}\right\},
\end{gathered}
$$

which are closed neighbourhoods of $z_{0}$ and $x_{0}$ respectively.

Choose $\alpha>0$ and set $W_{0}:=\bar{B}\left(w_{0}, \alpha\right)$.

Moreover, by assumption (iv), there exists $c \geq 0$ such that

$$
|\phi(v, x)| \leq c, \quad \forall(v, x) \in V \times X
$$

Thus, set $\delta:=\min \left\{\frac{2}{\rho\left(\alpha+\left|w_{0}\right|+c\right)}, \frac{\gamma_{2}}{2\left(\alpha+\left|w_{0}\right|+c\right)}\right\}$.

We prove that $\delta, U_{0}, V, W_{0}, X_{0}$ and $Z_{0}$ fulfill Hypotheses $1^{*}, 2^{*}, 3^{*}$ and $4^{*}$.

Let us show first that $P_{C}$ is a continuous selection of $\Pi_{X_{0}}$ on $U_{0} \times Z_{0}$.

As $U_{0} \subseteq\left\{u \in U \cap O\right.$ : haus $\left.\left(C(u), C\left(u_{0}\right)<\eta\right)\right\}$, and $C(\cdot)$ satisfies (i), (ii), and (iii) of Theorem 4.16, we have, by the same theorem, that $P_{C}$ is single-valued and continuous on $U_{0} \times Z_{0}$.

We also know by Theorem 4.16 that $P_{C(u)}(z) \in(N(C(u), \cdot)+I)^{-1}(z)$, for all $(u, z) \in U_{0} \times Z_{0}$.

It remains to show that $P_{C(u)}(z) \in X_{0}$ for all $(u, z) \in U_{0} \times Z_{0}$. Let $(u, z) \in U_{0} \times Z_{0}$. By definition of $P_{C}$ we have $P_{C(u)}(z) \in C(u)$. Therefore by definition of $U_{0}$ we obtain

$$
d_{C\left(u_{0}\right)}\left(P_{C(u)}(z)\right) \leq \text { haus }\left(C(u), C\left(u_{0}\right)\right)<\min \left\{\frac{\gamma_{2}}{2}, \gamma\right\}
$$

hence $P_{C(u)}(z) \in X_{0}$. Thus

$$
P_{C(u)}(z) \in(N(C(u), \cdot)+I)^{-1}(z) \cap X_{0}=\Pi_{X_{0}}(u, z), \quad \forall(u, z) \in U_{0} \times Z_{0},
$$

which shows that $P_{C}$ is a continuous selection of $\Pi_{X_{0}}$ on $U_{0} \times Z_{0}$.

We show now that for all $(v, w, x) \in V \times W_{0} \times X_{0}$,

$$
\zeta^{\delta}(v, w, x) \in Z
$$


Let $(v, w, x) \in V \times W_{0} \times X_{0}$. One has

$$
\begin{aligned}
d_{C\left(u_{0}\right)}\left(\zeta^{\delta}(v, w, x)\right) & =d_{C\left(u_{0}\right)}(x+\delta(w-\phi(v, x))) \\
& \leq d_{C\left(u_{0}\right)}(x)+|\delta(w-\phi(v, x))| \\
& \leq \frac{\gamma_{2}}{2}+\delta\left(\left|w-w_{0}\right|+\left|w_{0}\right|+c\right) \\
& \leq \frac{\gamma_{2}}{2}+\delta\left(\alpha+\left|w_{0}\right|+c\right) \\
& \leq \frac{\gamma_{2}}{2}+\frac{\gamma_{2}}{2}=\gamma_{2}
\end{aligned}
$$

hence $\zeta^{\delta}(v, w, x) \in Z$.

We show now that for all $(u, v, w, x) \in U_{0} \times V \times W_{0} \times X_{0}$ such that

$$
w \in N(C(u), x)+\phi(v, x),
$$

one has $x=P_{C(u)}\left(\zeta^{\delta}(v, w, x)\right)$.

Let $(u, v, w, x) \in U_{0} \times V \times W_{0} \times X_{0}$ such that $w \in N(C(u), x)+\phi(v, x)$. This implies that

$$
\delta(w-\phi(v, x)) \in N(C(u), x) .
$$

This gives that $x \in C(u)$. Further, by definition of $\delta$, one has

$$
\begin{aligned}
|\delta(w-\phi(v, x))| & \leq \delta\left(\left|w-w_{0}\right|+\left|w_{0}\right|+|\phi(v, x)|\right) \\
& \leq \delta\left(\alpha+\left|w_{0}\right|+c\right) \\
& \leq \frac{2}{\rho}
\end{aligned}
$$

we deduce that

$$
\frac{\rho}{2} \delta(w-\phi(v, x)) \in N(C(u), x) \cap \mathbb{B} .
$$

As $U_{0} \subseteq U$, the latter inclusion and Proposition 4.17 imply

$$
x \in P_{C(u)}\left(x+\frac{2}{\rho} \frac{\rho}{2} \delta(w-\phi(v, x))\right)=P_{C(u)}(x+\delta(w-\phi(v, x)))=P_{C(u)}\left(\zeta^{\delta}(v, w, x)\right) .
$$

Since $\zeta^{\delta}(v, w, x) \in Z_{0}$ with $P_{C}$ single-valued on $U_{0} \times Z_{0}$, we get that

$$
x=P_{C(u)}\left(\zeta^{\delta}(v, w, x)\right) .
$$

Finally, assumption (iv) gives directly that $\phi$ is continuous on $V \times X_{0}$ as $X_{0} \subseteq X$.

It only remains to show that $Z_{0}$ is a neighbourhood of $z_{0}:=\zeta^{\delta}\left(v_{0}, w_{0}, x_{0}\right)$ to conclude the proof. As

$$
P_{C\left(u_{0}\right)}\left(z_{0}\right)=P_{C\left(u_{0}\right)}\left(\zeta^{\delta}\left(v_{0}, w_{0}, x_{0}\right)\right)=x_{0},
$$

with $P_{C}$ continuous on $U_{0} \times Z_{0}$, one has that $P_{C}^{-1}\left(X_{0}\right) \subseteq U_{0} \times Z_{0}$ is a neighbourhood of $\left(u_{0}, z_{0}\right)$ since $X_{0}$ is a neighbourhood of $x_{0}$, hence $Z_{0}$ is a neighbourhood of $z_{0}$ which concludes the proof.

We are now ready to derive stability results under prox-regularity assumptions.

\subsection{Stability theorems under prox-regularity}

Let us recall the two problems considered in Section 3.

Given a solution $x_{0}$ of $\left(V I\left(u, v_{0}, w_{0}\right)\right)$, that is, $u_{0} \in \mathcal{U}, v_{0} \in \mathcal{V}, w_{0}, x_{0} \in \mathcal{H}$ verify the inclusion

$$
w_{0} \in N\left(C\left(u_{0}\right), x_{0}\right)+\phi\left(v_{0}, x_{0}\right),
$$

the first problem consists in studying the stability of $x$ under a variation of $w$, i.e., the stability of with respect to $w$ of the solution $x$ of the variational inequality

$$
w \in N\left(C\left(u_{0}\right), x\right)+\phi\left(v_{0}, x\right) .
$$


The second problem studies the stability of $x$ with respect to the pair $(u, v)$ of the inclusion

$$
w_{0} \in N(C(u), x)+\phi(v, x) .
$$

Dealing with (Prob1), we first adapt the definitions of section 3 to the new hypotheses setting.

Definition 4.19. Assuming $\delta>0, U:=\left\{u_{0}\right\} \subseteq \mathcal{U}, V:=\left\{v_{0}\right\} \subseteq \mathcal{V}$ and $W, X, Z \subseteq \mathcal{H}$ fulfill Hypotheses $1^{*}$, $2^{*}, 3^{*}$ and $4^{*}$, we define

$$
\begin{gathered}
\mathcal{X}^{*}=\left\{x \in X: \exists w \in W \text { s.t. } w \in N\left(C\left(u_{0}\right), x\right)+\phi\left(v_{0}, x\right) \text { and } \zeta^{\delta}\left(v_{0}, w, x\right) \in Z\right\}, \\
\mathcal{W}^{*}=\left\{w \in W: \exists x \in X \text { s.t. } w \in N\left(C\left(u_{0}\right), x\right)+\phi\left(v_{0}, x\right) \text { and } \zeta^{\delta}\left(v_{0}, w, x\right) \in Z\right\}, \\
\mathcal{Z}^{*}=\left\{z \in Z: \exists x \in X, \exists w \in W \text { s.t. } w \in N\left(C\left(u_{0}\right), x\right)+\phi\left(v_{0}, x\right) \text { and } z=\zeta^{\delta}\left(v_{0}, w, x\right)\right\} .
\end{gathered}
$$

and

$$
\begin{aligned}
M^{*}\left(u_{0}, v_{0}\right): \mathcal{W}^{*} & \rightrightarrows \mathcal{X}^{*} ., \\
w & \mapsto\left\{x \in X:\left(u_{0}, v_{0}, w, x\right) \in \mathcal{M}^{*}\left(u_{0}, v_{0}\right)\right\}
\end{aligned}
$$

where $\mathcal{M}^{*}\left(u_{0}, v_{0}\right)=\mathcal{M}^{*}$ and $\mathcal{N}^{*}\left(u_{0}, v_{0}\right)=\mathcal{N}^{*}$.

We can now state a result similar to Theorem 3.5 but under prox-regular assumptions.

Theorem 4.20. Let $u_{0} \in \mathcal{U}, v_{0} \in \mathcal{V}, w_{0}, x_{0} \in \mathcal{H}$ be such that

$$
w_{0} \in N\left(C\left(u_{0}\right), x_{0}\right)+\phi\left(v_{0}, x_{0}\right) \text {. }
$$

\section{Suppose}

(i) the set $C\left(u_{0}\right)$ is uniformly prox-regular;

(ii) $\phi\left(v_{0}, \cdot\right)$ is bounded and continuous on $\left\{x \in \mathcal{H}: d_{C\left(u_{0}\right)}(x) \leq \gamma\right\}$ for some $\gamma>0$.

Then there exist $\delta>0, W$ neighbourhood of $w_{0}, X$ neighbourhood of $x_{0}$ and $Z$ neighbourhood of $z_{0}$ such that $\delta, U:=\left\{u_{0}\right\}, V:=\left\{v_{0}\right\}, W, X$ and $Z$ fulfill Hypotheses $1^{*}, 2^{*}, 3^{*}$ and $4^{*}$.

Therefore, considering $\mathcal{M}^{*}\left(u_{0}, v_{0}\right), \mathcal{N}^{*}\left(u_{0}, v_{0}\right), \mathcal{X}^{*}, \mathcal{W}^{*}, \mathcal{Z}^{*}$ and $M^{*}\left(u_{0}, v_{0}\right)$ as in Definition 4.19, one has that the map $f_{X}^{*}\left(u_{0}, v_{0}, \cdot\right): \mathcal{Z}^{*} \rightarrow \mathcal{W}^{*}$ is an homeomorphism if and only if the set-valued mapping $M^{*}\left(u_{0}, v_{0}\right)$ is single-valued on $\mathcal{W}^{*}$ and its associated mapping $m$ is continuous on $\mathcal{W}^{*}$.

In this case, one has for all $w \in \mathcal{W}^{*}$,

$$
m(w)=\pi_{X}\left(u_{0}, f_{X}^{*}\left(u_{0}, v_{0}, \cdot\right)^{-1}(w)\right)
$$

and

$$
f_{X}^{*}\left(u_{0}, v_{0}, \cdot\right)^{-1}(w)=\zeta^{\delta}\left(u_{0}, v_{0}, m(w)\right)
$$

Further

$$
\mathcal{M}^{*}\left(u_{0}, v_{0}\right)=\left\{\left(u_{0}, v_{0}\right)\right\} \times\left\{(w, m(w)): w \in \mathcal{W}^{*}\right\}
$$

and

$$
\mathcal{N}^{*}\left(u_{0}, v_{0}\right)=\left\{\left(u_{0}, v_{0}\right)\right\} \times\left\{\left(w, f_{X}^{*}\left(u_{0}, v_{0}, \cdot\right)^{-1}(w)\right): w \in \mathcal{W}\right\} .
$$

Proof. Let us consider the constant set-valued mapping

$$
\begin{aligned}
S: \mathcal{U} & \rightrightarrows \mathcal{H} \\
u & \mapsto C\left(u_{0}\right) .
\end{aligned}
$$

As the set $C\left(u_{0}\right)$ is uniformly prox-regular, say for the paramater $\rho>0$, it is obvious that the set valuedmapping $S(\cdot)$ is (uniformly) prox-regular with compatible parametrisation by $u$ at $u_{0}$ for the parameters $\mathcal{U}$ and $\rho>0$. Likewise, it is straightforward that $S(\cdot)$ is Hausdorff and Attouch-Wets continuous on $\mathcal{U}$.

Now let us define the mapping

$$
\begin{aligned}
\Phi: \mathcal{V} \times \mathcal{H} & \rightarrow \mathcal{H} . \\
(v, x) & \mapsto \phi\left(v_{0}, x\right)
\end{aligned}
$$

Then by the assumption on $\phi\left(v_{0}, \cdot\right)$, it is easy to check that $\Phi$ is continuous and bounded on $\mathcal{V} \times\{x \in \mathcal{H}$ : $\left.d_{C\left(u_{0}\right)}(x) \leq \gamma\right\}$.

We can then apply Theorem 4.18 to obtain the existence of $\delta>0, U_{0}$ neighbourhood of $u_{0}, V_{0}$ neighbourhood of $v_{0}, W$ neighbourhood of $w_{0}, X$ neighbourhood of $x_{0}$ and $Z$ neighbourhood of $\zeta^{\delta}\left(v_{0}, w_{0}, x_{0}\right)$ such that 
(a) the set-valued mapping $(u, z) \mapsto(N(S(u), \cdot)+I)^{-1}(z) \cap X$ as a continuous selection $\pi_{X}$ on $U_{0} \times Z$;

(b) $x+\delta(w-\Phi(v, x)) \in Z$ for all $(v, w, x) \in V_{0} \times W \times X$;

(c) $x=\pi_{X}\left(u, \zeta^{\delta}(v, w, x)\right)$ for all $(u, v, w, x) \in U_{0} \times V_{0} \times W \times X$ such that $w \in N(S(u), x)+\Phi(v, x)$;

(d) $\Phi$ is continuous on $V_{0} \times X$.

Then, setting $U:=\left\{u_{0}\right\}$ and $V:=\left\{v_{0}\right\}$, we get by (a) that $(u, z) \mapsto(N(C(u), \cdot)+I)^{-1}(z) \cap X$ is a continuous selection $\pi_{X}$ on $U \times Z$, since $C(u)=S(u)$ for all $u \in U$.

Likewise, as $\Phi(v, x)=\phi(v, x)$ for all $(v, x) \in V \times X$, we obtain that

$$
x+\delta(w-\phi(v, x)) \in Z, \quad \forall(v, w, x) \in V \times W \times X .
$$

Now taking $(u, v, w, x) \in U \times V \times W \times X$ such that $w \in N(C(u), x)+\phi(v, x)$, we have $w \in N(S(u), x)+$ $\Phi(v, x)$, hence $x=\pi_{X}\left(u, \zeta^{\delta}(v, w, x)\right)$

And finally, it is straightforward that $\phi$ is continuous on $U \times X$.

This gives that $\delta, U:=\left\{u_{0}\right\}, V:=\left\{v_{0}\right\}, W, X$ and $Z$ fulfill Hypotheses $1^{*}, 2^{*}, 3^{*}$ and $4^{*}$.

We can then consider $\mathcal{M}^{*}\left(u_{0}, v_{0}\right), \mathcal{N}^{*}\left(u_{0}, v_{0}\right), \mathcal{X}^{*}, \mathcal{W}^{*}, \mathcal{Z}^{*}$ and $M^{*}\left(u_{0}, v_{0}\right)$ as in Definition 4.19 and follow the proof of Theorem 3.5 to obtain the result.

Concerning Problem (Prob2), we also need to introduice the following notations.

Definition 4.21. Assuming $\delta>0, U \subseteq \mathcal{U}, V \subseteq \mathcal{V}, W=\left\{w_{0}\right\} \subseteq \mathcal{H}$ and $X, Z \subseteq \mathcal{H}$ fulfill Hypotheses $1^{*}, 2^{*}$, $3^{*}$ and $4^{*}$, we define the set-valued mappings

$$
\begin{aligned}
& M^{*}\left(w_{0}\right): \quad U \times V \quad \rightrightarrows \quad X \\
& (u, v) \mapsto\left\{x \in X:\left(u, v, w_{0}, x\right) \in \mathcal{M}^{*}\left(w_{0}\right)\right\}
\end{aligned}
$$

and

$$
\begin{aligned}
& N^{*}\left(w_{0}\right): \quad U \times V \quad \rightrightarrows Z \\
& (u, v) \mapsto\left\{z \in Z:\left(u, v, w_{0}, z\right) \in \mathcal{N}^{*}\left(w_{0}\right)\right\}
\end{aligned}
$$

where $\mathcal{M}^{*}\left(w_{0}\right)=\mathcal{M}^{*}, \mathcal{N}^{*}\left(w_{0}\right)=\mathcal{N}^{*}$ and $\mathcal{L}^{*}\left(w_{0}\right)=\mathcal{L}^{*}$.

Since the proof of Lemma 3.6 is only based on the homeomorphy result which still holds when dealing with the relaxed hypotheses, we have the following lemma.

Lemma 4.22. Assume $\delta>0, U \subseteq \mathcal{U}, V \subseteq \mathcal{V}, W=\left\{w_{0}\right\} \subseteq \mathcal{H}$ and $X, Z \subseteq \mathcal{H}$ fulfill Hypotheses $1^{*}$, 2*, 3* and $4^{*}$, and define $\mathcal{M}^{*}\left(w_{0}\right), \mathcal{N}^{*}\left(w_{0}\right), M^{*}\left(w_{0}\right)$ and $N^{*}\left(w_{0}\right)$ as above.

Considering $\left(u_{0}, v_{0}\right) \in U \times V$, we have that $M^{*}\left(w_{0}\right)$ is lower semicontinuous at $\left(u_{0}, v_{0}\right)$ if and only if $N^{*}\left(w_{0}\right)$ is lsc at $\left(u_{0}, v_{0}\right)$.

Proof. Similar to the proof of Lemma 3.6.

We can now give the stability theorems for Problem (Prob2) under prox-regularity assumption. Remark that, as the neighbourhood $X$ of $x_{0}$ and $Z$ of $z_{0}$ need to be bounded to apply the degree theory, we need to assume that $C\left(u_{0}\right)$ is bounded, since the neighbourhoods $X$ and $Z$ of $x_{0}$ and $z_{0}$ resp. created in Theorem 4.18 are of the form

$$
\left\{x \in \mathcal{H}: d_{C\left(u_{0}\right)}(x)<\gamma\right\},
$$

with $\gamma>0$.

Theorem 4.23. Let $u_{0} \in \mathcal{U}, v_{0} \in \mathcal{V}, w_{0}, x_{0} \in \mathcal{H}$ such that

$$
w_{0} \in N\left(C\left(u_{0}\right), x_{0}\right)+\phi\left(v_{0}, x_{0}\right) .
$$

\section{Suppose}

(i) $C(\cdot)$ is uniformly prox-regular at $u_{0}$;

(ii) $C(\cdot)$ is Attouch-Wets continuous on a neighbourhood $O$ of $u_{0}$; 
(iii) $C\left(u_{0}\right)$ is bounded and $C(\cdot)$ is Hausdorff continuous at $u_{0}$;

(iv) $\phi$ is continuous on $V \times\left\{x \in \mathcal{H}: d_{C\left(u_{0}\right)}(x) \leq \gamma\right\}$ for some neighbourhood $V$ of $v_{0}$ and some $\gamma>0$

Then there exist $\delta, U$, resp. $V, X$ and $Z$, neighbourhoods of $u_{0}$, resp. $v_{0}, x_{0}$ and $z_{0}:=\zeta\left(v_{0}, w_{0}, x_{0}\right)$ such that $\delta, U, V, W:=\left\{w_{0}\right\}, X$ and $Z$ fulfill Hypotheses $1^{*}, 2^{*}, 3^{*}$ and $4^{*}$ and we can consider $\mathcal{M}^{*}\left(w_{0}\right), \mathcal{N}^{*}\left(w_{0}\right)$ and $\mathcal{L}^{*}\left(w_{0}\right)$, and, $M^{*}\left(w_{0}\right)$ and $N^{*}\left(w_{0}\right)$ as above.

Suppose in addition that:

(1)

$$
\begin{aligned}
& \pi_{X}\left(\cdot, \zeta^{\delta}\left(\cdot, w_{0}, \cdot\right)\right): \quad U \times V \times X \quad \rightarrow \quad \mathcal{H} \\
& (u, v, x) \quad \mapsto \quad \pi_{X}\left(u, \zeta^{\delta}\left(v, w_{0}, x\right)\right)
\end{aligned}
$$

is with finite dimensional range;

(2) $M\left(w_{0}\right)\left(u_{0}, v_{0}\right)=\left\{x_{0}\right\}$;

(3) $\operatorname{deg}_{\mathcal{H}}\left(g_{X}^{*}\left(u_{0}, v_{0}, w_{0}, \cdot\right)\right.$, int $\left.X, 0\right) \neq 0$.

Then

(a) $M^{*}\left(w_{0}\right)\left(u_{0}, v_{0}\right)=\left\{x_{0}\right\}$ and $N^{*}\left(w_{0}\right)\left(u_{0}, v_{0}\right)=\left\{z_{0}\right\}$;

(b) there exist a neighbourhood $U_{0} \subseteq U$ of $u_{0}$ and a neighbourhood $V_{0} \subseteq V$ of $v_{0}$ such that

$$
M^{*}\left(w_{0}\right)(u, v) \neq \emptyset \quad \text { and } \quad N^{*}\left(w_{0}\right)(u, v) \neq \emptyset, \quad \forall(u, v) \in U_{0} \times V_{0} ;
$$

(c) $M^{*}\left(w_{0}\right)$ and $N^{*}\left(w_{0}\right)$ are lsc at $\left(u_{0}, v_{0}\right)$.

Proof. By assumptions (i), (ii), (iii) and (iv) we can apply Theorem 4.18 to get the existence of $\delta, U$, resp. $V$, $X$ and $Z$, neighbourhoods of $u_{0}$, resp. $v_{0}, x_{0}$ and $z_{0}:=\zeta\left(v_{0}, w_{0}, x_{0}\right)$ such that $\delta, U, V, W:=\left\{w_{0}\right\}, X$ and $Z$ fulfill Hypotheses $1^{*}, 2^{*}, 3^{*}$ and $4^{*}$.

Now in order to follow the proof of Theorem 3.7, it only remains to see that $X$ is bounded since we know that $X$ is closed. This is given by the fact that $C\left(u_{0}\right)$ is bounded. Indeed, we know that the neighbourhood $X$ of $x_{0}$ created in the proof of Theorem 4.18 is of the form $\left\{x \in \mathcal{H}: d_{C\left(u_{0}\right)}(x)<\gamma_{1}\right\}$ with $\gamma_{1}>0$, so $X$ is bounded since $C\left(u_{0}\right)$ is bounded.

We get the result by following the proof of Theorem 3.7.

Likewise, we express Theorem 3.8 in the prox-regular setting.

Theorem 4.24. Let $u_{0} \in \mathcal{U}, v_{0} \in \mathcal{V}, w_{0}, x_{0} \in \mathcal{H}$ such that

$$
w_{0} \in N\left(C\left(u_{0}\right), x_{0}\right)+\phi\left(v_{0}, x_{0}\right) .
$$

Suppose

(i) $C(\cdot)$ is uniformly prox-regular at $u_{0}$;

(ii) $C(\cdot)$ is Attouch-Wets continuous on a neighbourhood $O$ of $u_{0}$;

(iii) $C\left(u_{0}\right)$ is bounded and $C(\cdot)$ is Hausdorff continuous at $u_{0}$;

(iv) $\phi$ is continuous on $V \times\left\{x \in \mathcal{H}: d_{C\left(u_{0}\right)}(x) \leq \gamma\right\}$ for some neighbourhood $V$ of $v_{0}$ and some $\gamma>0$

Then there exist $\delta, U$, resp. $V, X$ and $Z$, neighbourhoods of $u_{0}$, resp. $v_{0}, x_{0}$ and $z_{0}:=\zeta\left(v_{0}, w_{0}, x_{0}\right)$ such that $\delta, U, V, W:=\left\{w_{0}\right\}, X$ and $Z$ fulfill Hypotheses $1^{*}, 2^{*}, 3^{*}$ and $4^{*}$ and we can consider $\mathcal{M}^{*}\left(w_{0}\right), \mathcal{N}^{*}\left(w_{0}\right)$ and $\mathcal{L}^{*}\left(w_{0}\right)$, and, $M^{*}\left(w_{0}\right)$ and $N^{*}\left(w_{0}\right)$ be as above.

Suppose in addition that:

$$
\begin{array}{cl}
U \times V \times Z & \rightarrow \mathcal{H} \\
(u, v, z) & \mapsto \pi_{X}(u, z)-\phi\left(v, \pi_{X}(u, z)\right)
\end{array}
$$

is with finite dimensional range; 
(2) $N\left(w_{0}\right)\left(u_{0}, v_{0}\right)=\left\{z_{0}\right\}$;

(3) $\operatorname{deg}_{\mathcal{H}}\left(f_{X}\left(u_{0}, v_{0}, \cdot\right)\right.$, int $\left.Z, w_{0}\right) \neq 0$.

Then

(a) $M^{*}\left(w_{0}\right)\left(u_{0}, v_{0}\right)=\left\{x_{0}\right\}$ and $N^{*}\left(w_{0}\right)\left(u_{0}, v_{0}\right)=\left\{z_{0}\right\}$;

(b) there exist a neighbourhood $U_{0} \subseteq U$ of $u_{0}$ and a neighbourhood $V_{0} \subseteq V$ of $v_{0}$ such that

$$
M^{*}\left(w_{0}\right)(u, v) \neq \emptyset \quad \text { and } \quad N^{*}\left(w_{0}\right)(u, v) \neq \emptyset, \quad \forall(u, v) \in U_{0} \times V_{0} ;
$$

(c) $M^{*}\left(w_{0}\right)$ and $N^{*}\left(w_{0}\right)$ are lsc at $\left(u_{0}, v_{0}\right)$.

Proof. By the arguments of the proof of Theorem 4.23 and following the proof of Theorem 3.8.

This concludes our study of stability of variational inequalities in the prox-regular setting.

\section{References}

[1] H. Attouch and R.J.-B. Wets, Isometries for the Legendre-Fenchel transform, Trans. Amer. Math. Soc. 296 (1986), 33-60.

[2] A. Canino, On p-convex sets and geodesics, J. Differential Equations 75 (1988), 118-157.

[3] F.H. Clarke, R.J. Stern and P.R. Wolenski, Proximal smoothness and the lower $C^{2}$ property, J. Convex Analysis 2 (1995), 117-144.

[4] G. Colombo and V. Goncharov, Variational inequalities and regularity properties of closed sets in Hilbert spaces, J. Convex Anal. 8 (2001), 197-221.

[5] G. Colombo and L. Thibault, Prox-regular sets and applications, to appear.

[6] J. Droniou, degr $\tilde{A}(\mathcal{C}$ s topologique et applications, Polycopi $\tilde{A}(\subset)$ gm3, http://www-gm3.univ-mrs.fr/polys.

[7] H. Federer, Curvature measures, Trans. Amer. Math. Soc. 93 (1959), 418-491.

[8] I. Fonseca and W. Gangbo, Degree theory in analysis and applications, Oxford science publications (1995).

[9] P.T. Harker and J.-S. Pang, Finite-dimensional variational inequality and nonlinear complementarity problems: a survey of theory, algorithms and applications, Math. Programming 48 (1990), 161-220.

[10] B.T. Kien and J.-C. Yao, Localization of generalized normal maps and stability of variational inequalities in reflexive Banach spaces, Set-Valued Anal. 16 (2008), 399-412.

[11] A. Levi, R.A. Poliquin and R.T. Rockafellar, Stability of locally optimal solutions, SIAM J. Optim. 10 (2000), 580-604.

[12] B.S. Mordukhovich, Variational Analysis and generalized differentiation, I: Basic theory, Springer Verlag, New-York, (2006).

[13] R.A. Poliquin and R.T. Rockafellar, Prox-regular functions in variational analysis, Trans. Amer. Math. Soc. 348 (1996), 1805-1838.

[14] R.A. Poliquin, R.T. Rockafellar and L. Thibault, Local differentiability of distance functions, Trans. Amer. Math. Soc. 352 (2000), no. 11, 5231-5249.

[15] R.T. Rockafellar and R.J.-B. Wets, Variational Analysis, Springer Verlag, New-York, (1998).

[16] S.M. Robinson, Sensitivity analysis of variational inequalities by normal-map techniques, Variational inequalities and network equilibrium problems (Erice, 1994), 257-269, Plenum, New York, 1995. 
[17] S.M. Robinson, Nonsmooth continuation for generalized equations Recent advances in optimization (Trier, 1996), 282-291, Lecture Notes in Econom. and Math. Systems, 452, Springer, Berlin, 1997.

[18] S.M. Robinson, Localized normal maps and the stability of variational conditions, Set-Valued Anal. 12 (2004), 259-274.

[19] S.M. Robinson, Aspects of the projector on prox-regular sets, Variational Analysis and Applications, Nonconvex Optim. Appl. 79, Springer, New York (2005), 963-973.

[20] A. Shapiro, Existence and differentiability of metric projections in Hilbert spaces, SIAM J. Optimization 4 (1994), 130-141.

[21] M. Sebbah and L. Thibault, Metric projection and compatibly parameterized families of prox-regular sets in Hilbert space, Nonlinear Anal. 75 (2012), 1547-1562.

[22] J.-P. Vial, Strong and weak convexity of sets and functions, Math. Oper. Res. 8 (1983), 231-259. 